Supporting Information for

\title{
Monolithic Chiral Nematic Organization of Cellulose Nanocrystals under Capillary Confinement
}

\author{
Minkyu Kim, ${ }^{a}$ Kellina Pierce, ${ }^{a}$ Michelle Krecker, ${ }^{a}$ Daria Bukharina, ${ }^{a}$ Katarina Adstedt, ${ }^{a}$ Dhriti \\ Nepal, ${ }^{b}$ Timothy Bunning, ${ }^{b}$ Vladimir V. Tsukruk ${ }^{a *}$ \\ ${ }^{a}$ School of Materials Science and Engineering, Georgia Institute of Technology, Atlanta, GA \\ 30332, USA \\ b Air Force Research Laboratory, Wright-Patterson Air Force Base, Ohio 45433, USA \\ * Corresponding Author E-mail: vladimir@mse.gatech.edu
}

Video S1: Accelerated video shows the CNC/TOPA/PEG film real-time formation with evaporation of water in the rectangular capillary $(0.400 \mathrm{~mm}$ width, $50 \mathrm{~mm}$ in length and gap thickness of $40 \mu \mathrm{m}$ ) under cross-polarized optical microscopy. The video presents last stages of drying, from 7 hours 53 minutes to 8 hours 25 minutes after the initiation of the process.

Video S2: Accelerated video shows the CNC/TOPA/PEG film real-time formation with evaporation of water in the round glass (VitroTubes $\left.{ }^{\mathrm{TM}}\right)$ capillaries $(0.6 \mathrm{~mm}$ inner diameter, $6 \mathrm{~cm}$ in length, wall thickness $0.12 \mathrm{~mm}$ ) under cross-polarized optical microscopy. The video presents last stages of drying. Videos are taken over 2.5 hours and film dried after 6 hours from start. 


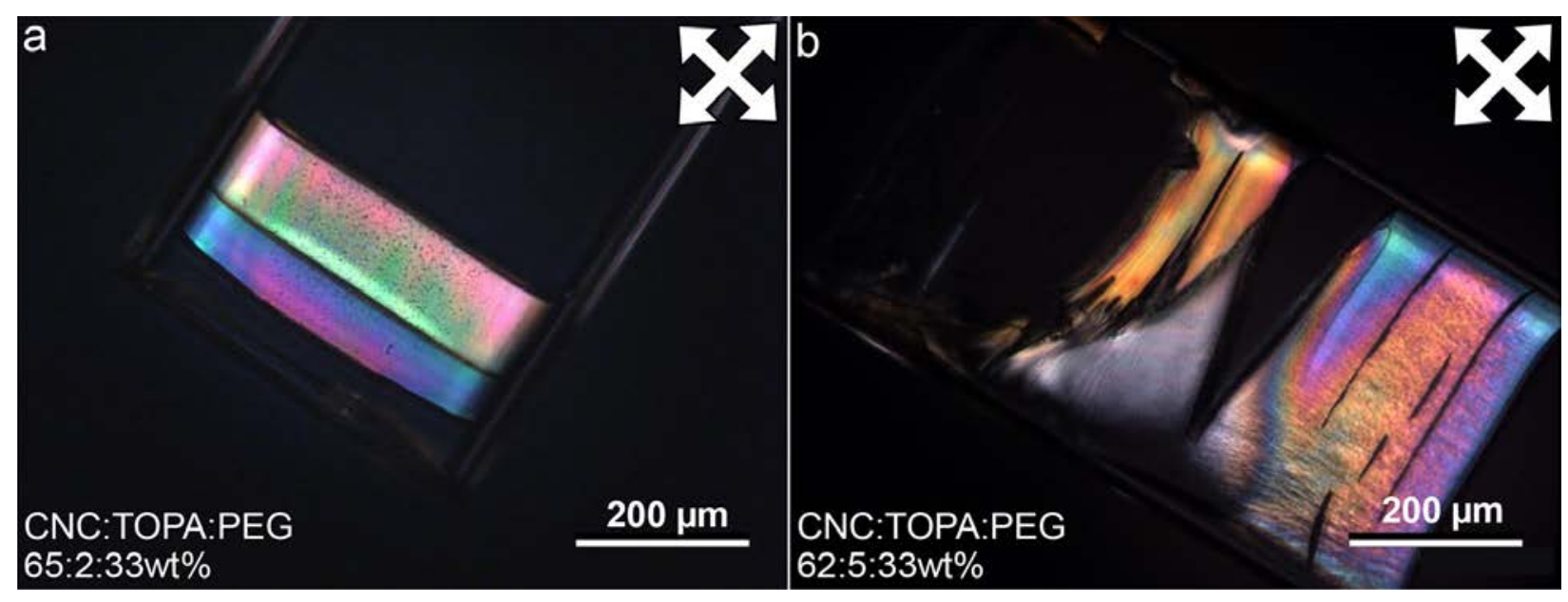

Figure S1. Cross-polarized optical microscopy images of (a) 65:2:33wt\% and (b) 62:5:33wt\% ratio CNC/TOPA/PEG film, that confirms that the best performance that prevents the cracks was achieved from a 64:3:33wt\% ratio CNC/TOPA/PEG film presented in the main text (Figure 2c). 


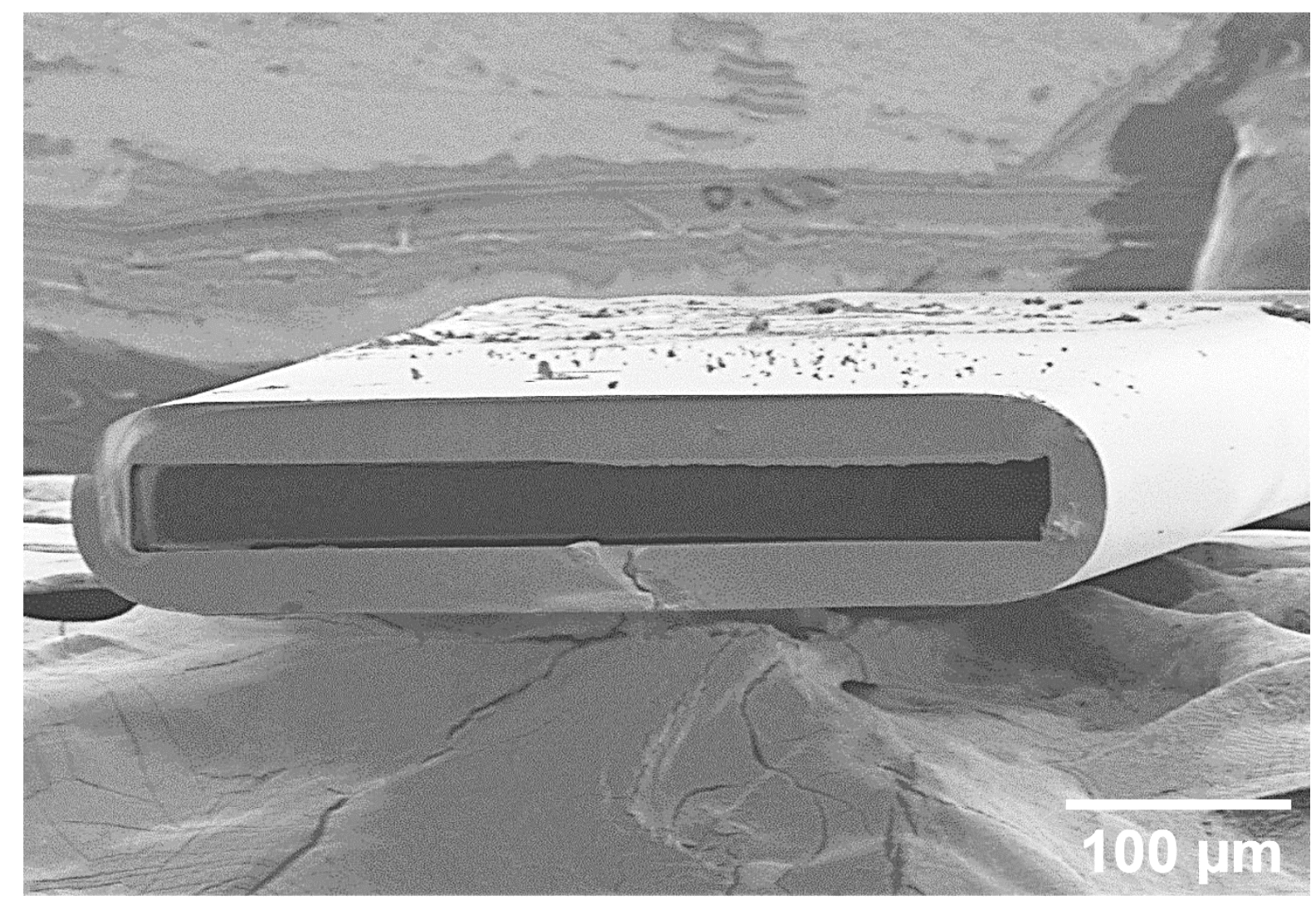

Figure S2. SEM image of CNC/TOPA/PEG film formed in the rectangular capillary. The thickness of film is $33 \mu \mathrm{m}$. 

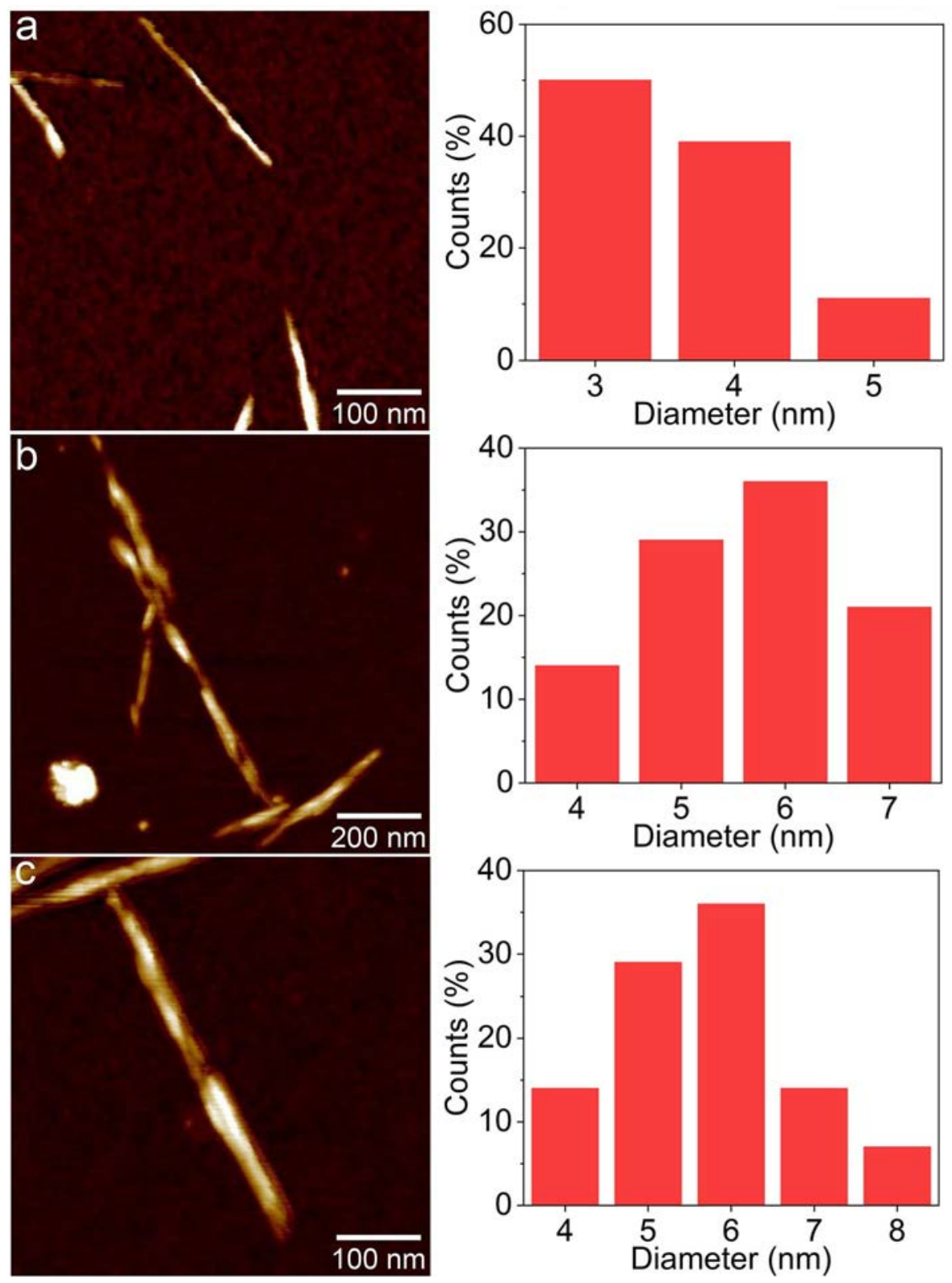

Figure S3. AFM topography image (left) and their diameter distribution (right) of individual nanocrystals from (a) CNC, (b) CNC/TOPA, and (c) CNC/TOPA/PEG suspensions. 

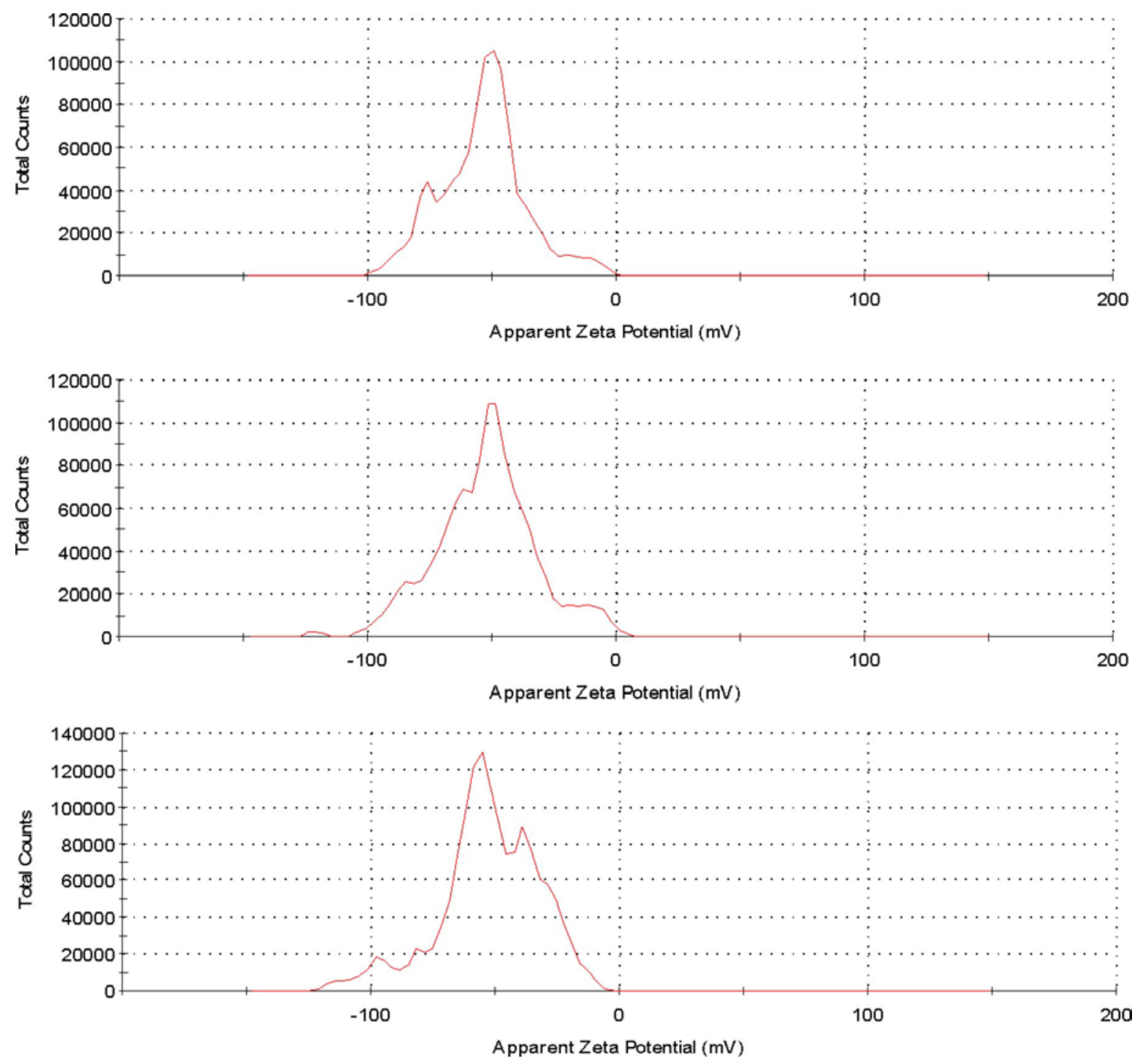

Figure S4. Zeta potential of CNC. 

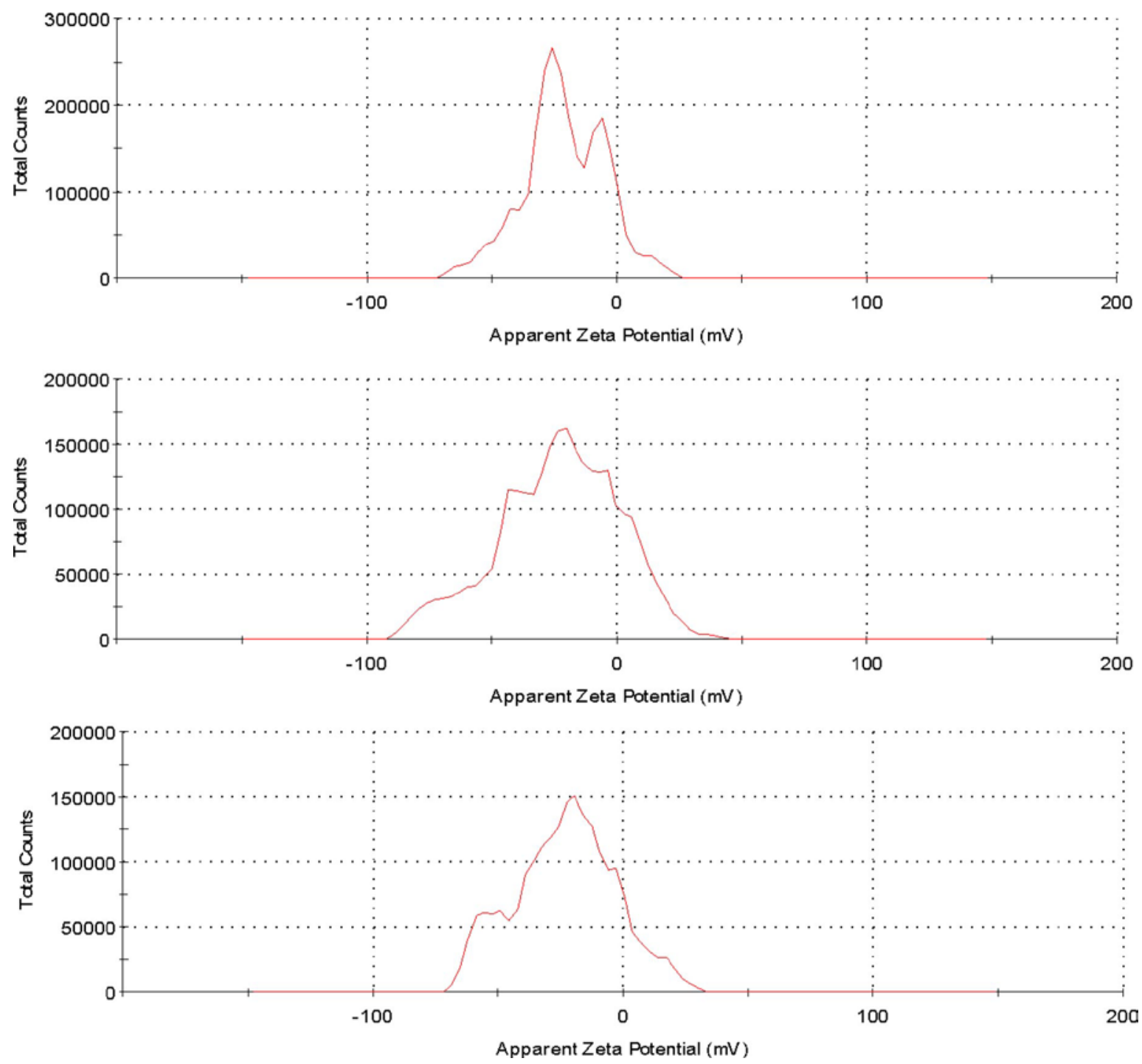

Figure S5. Zeta potential of CNC/TOPA. 

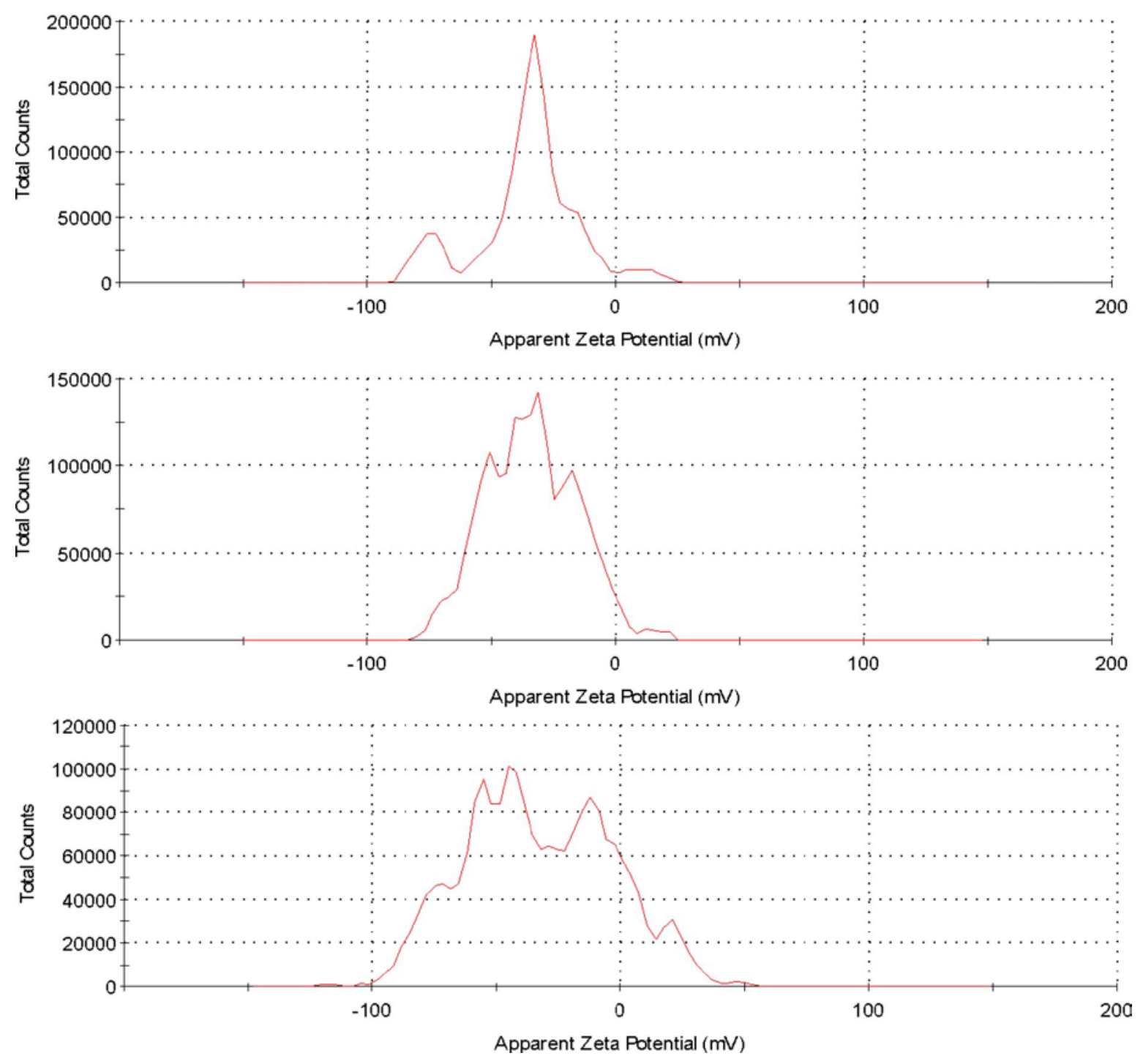

Figure S6. Zeta potential of CNC/TOPA/PEG. 

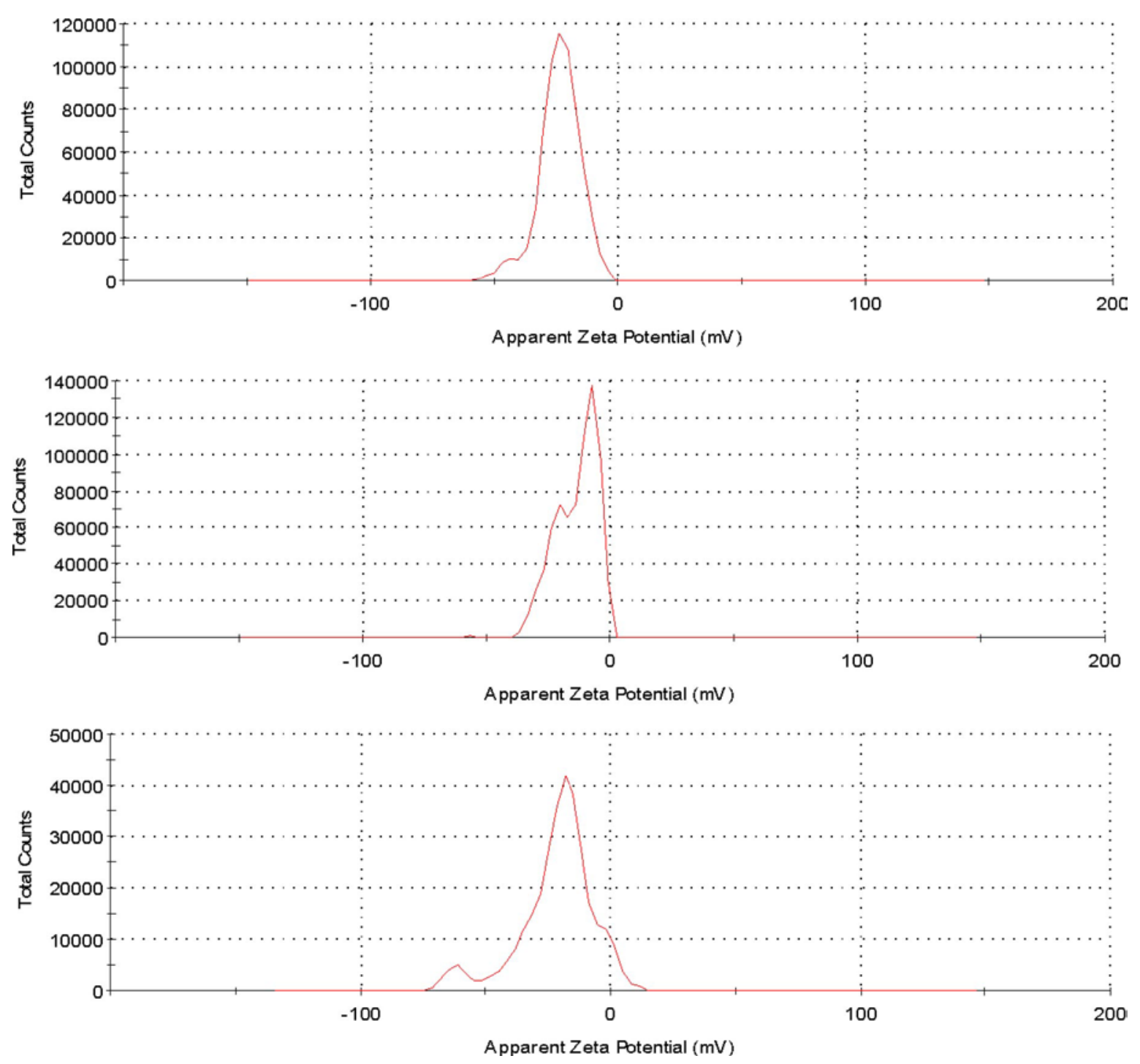

Figure S7. Zeta potential of PEG.

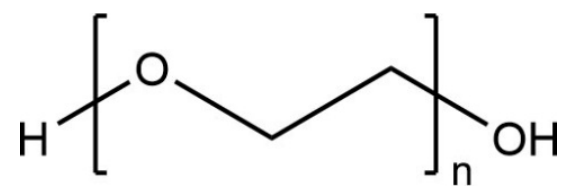

Figure S8. Chemical structure of PEG utilized in this research. 


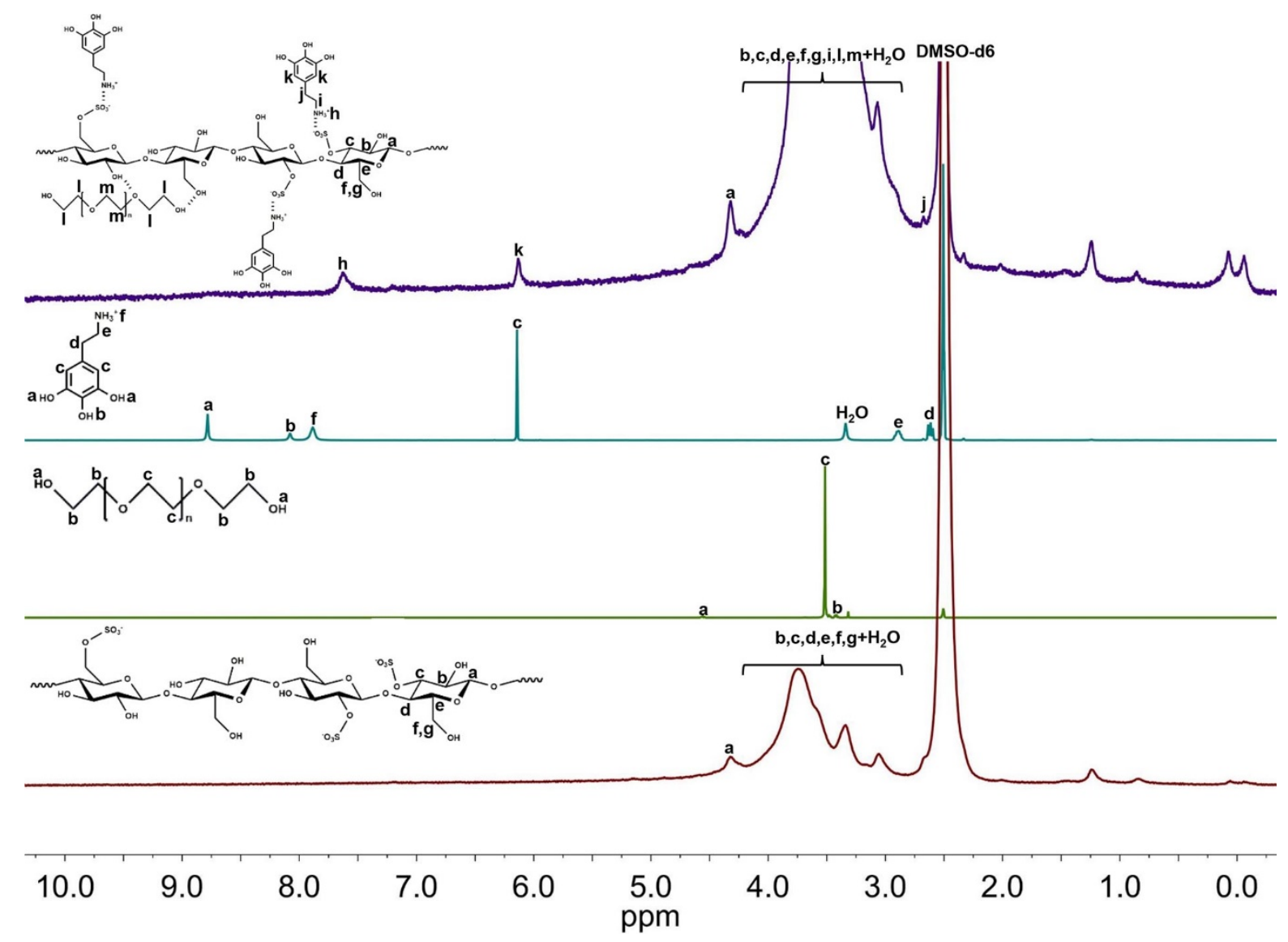

Figure S9. Proton NMR of CNC/TOPA/PEG, TOPA, PEG, and CNC, respectively. ${ }^{1} \mathrm{H}$ NMR spectra confirms that CNC/TOPA/PEG is successfully synthesized by illustrating the proton peaks of CNC, TOPA, and PEG. ${ }^{1,2}$ Additionally, ${ }^{1} \mathrm{H}$ NMR spectrum of CNC/TOPA/PEG shows that TOPA exists as protonated form in the solvent, demonstrating that the positively charged protonated amine ion of TOPA bonds with negatively charged sulfonate groups from CNC via the ion-ion interaction. 


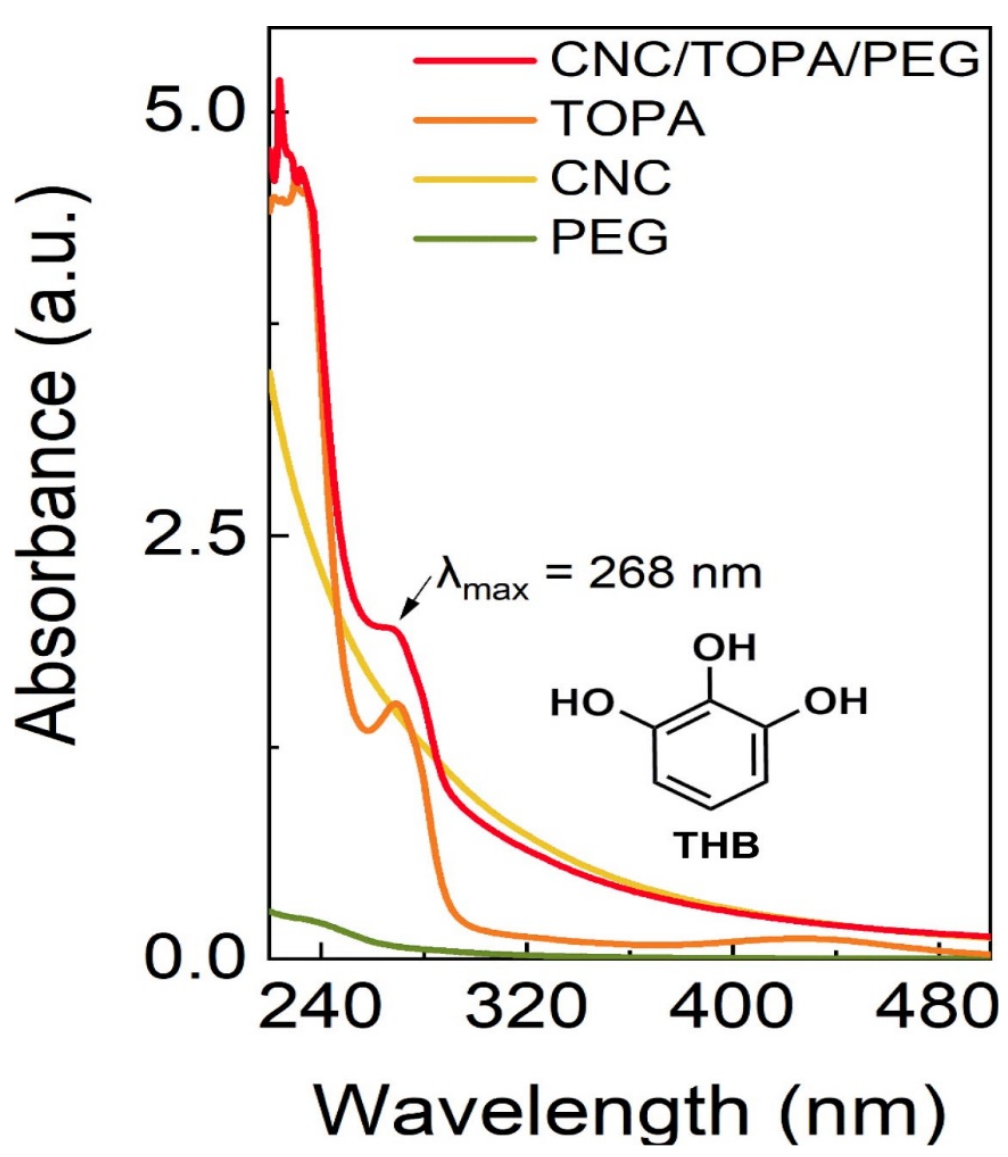

Figure S10. UV-vis spectra of CNC/TOPA/PEG, TOPA, CNC, and PEG, respectively. The CNC/TOPA/PEG exhibits the intrinsic pyrogallol absorption peak at $268 \mathrm{~nm}$ ascribed from TOPA moiety in CNC/TOPA/PEG suspension ${ }^{3}$, representing the successful incorporation of TOPA molecules in CNC/TOPA/PEG. 


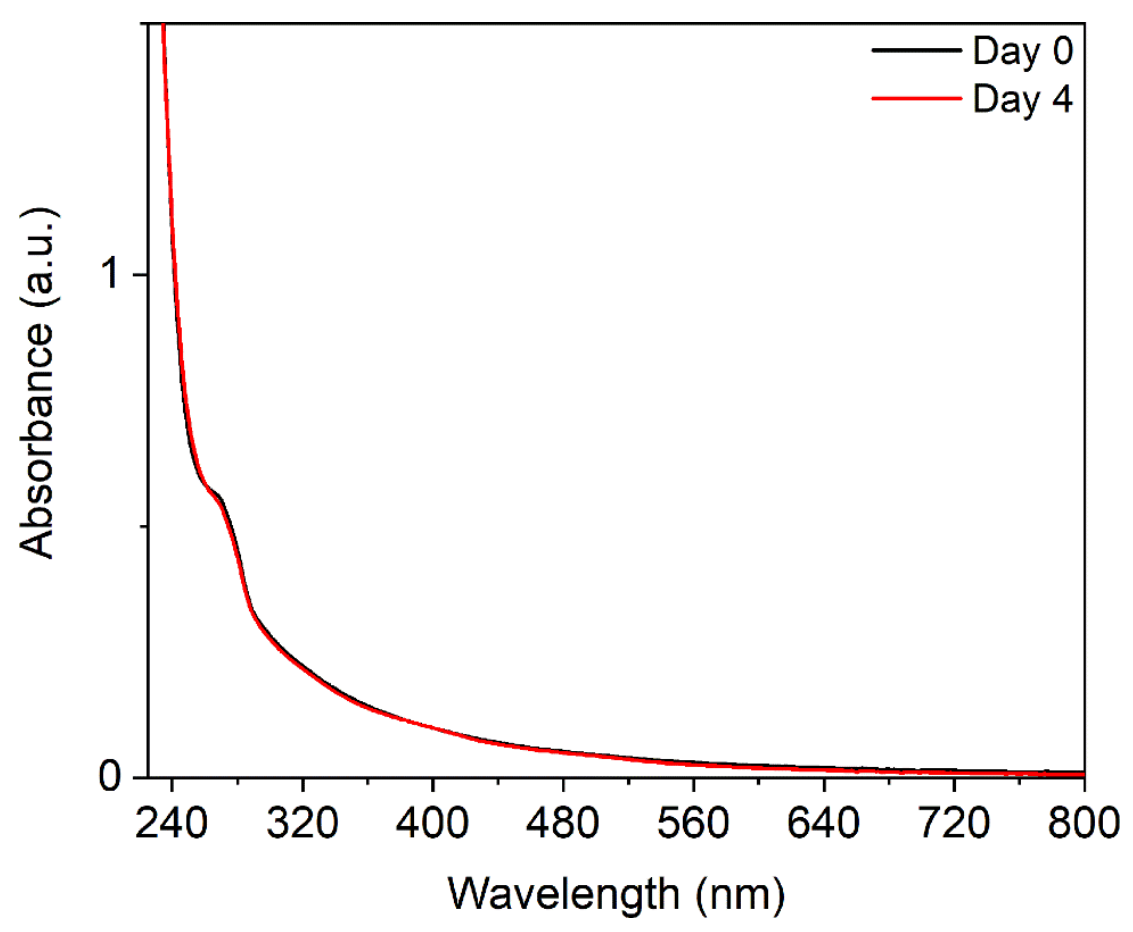

Figure S11. UV-vis spectra of CNC/TOPA/PEG suspension at day 0 and 4 with exposure to air under room light after 24h stirring of CNC/TOPA/PEG suspension after addition of PEG.

The CNC/TOPA/PEG suspension displays only pyrogallol absorption peak at $268 \mathrm{~nm}$ attributed to TOPA moiety even after 4 days of the suspension-exposure to air under room light. It has been reported that when the gallol group is oxidatively polymerized, the polymer possesses galloquinone structures ${ }^{4}$ that exhibits the absorbance peak at $320 \mathrm{~nm}^{5}$, $370 \mathrm{~nm}, 400 \mathrm{~nm}^{6}$, or 420 $\mathrm{nm}^{6}$ in the UV-vis spectrum. Therefore, this result confirms that the TOPA moiety is not oxidized and not polymerized in the suspension when the suspension is exposed to the air for 4 days under room light. 


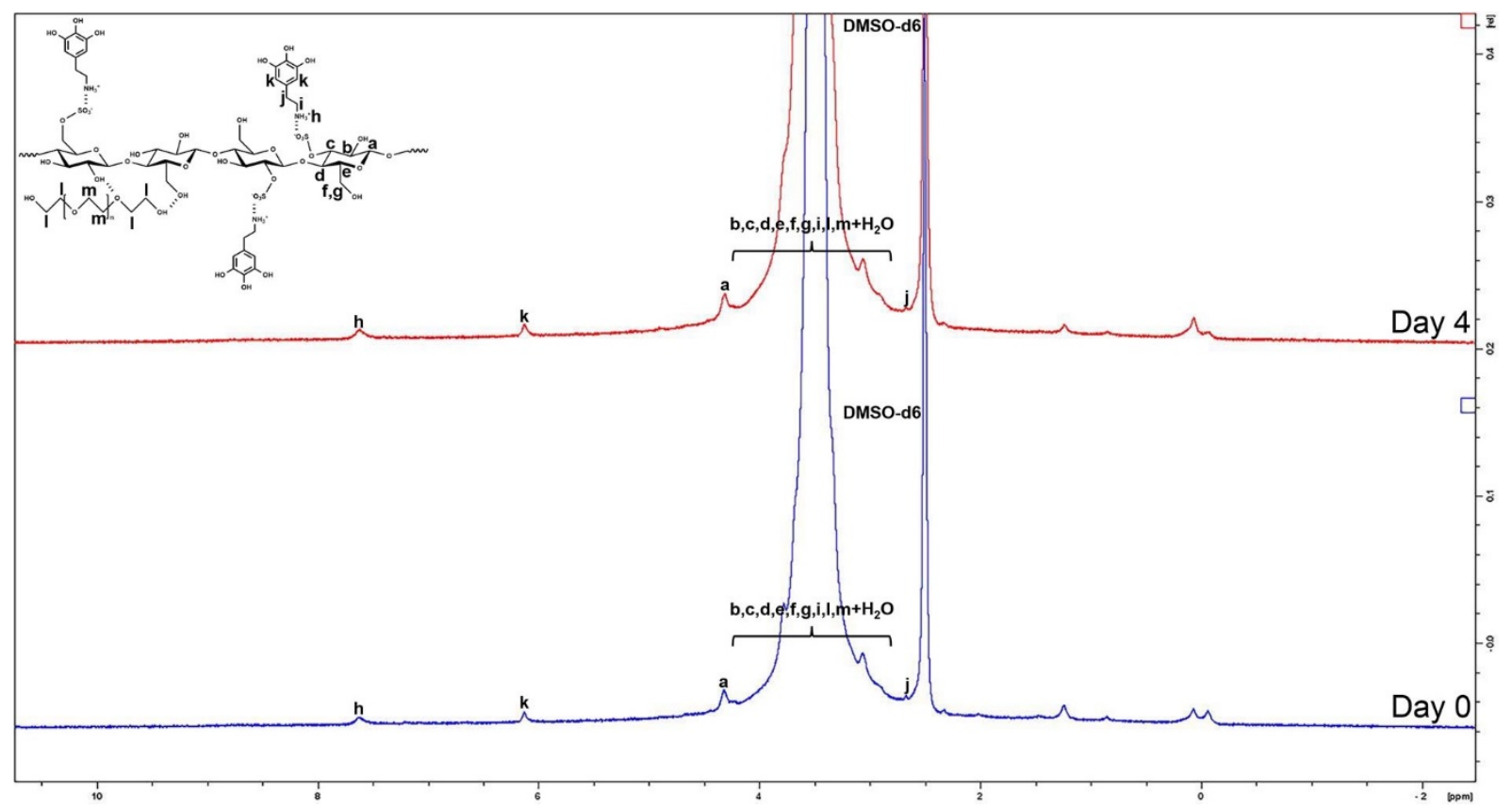

Figure S12. Proton NMR of CNC/TOPA/PEG suspension at day 0 and 4 with exposure to air under room light after 24h stirring of CNC/TOPA/PEG suspension after addition of PEG.

New proton peaks have not been observed for CNC/TOPA/PEG suspension after 4 days of the exposure to air under room light, indicating new chemicals have not been generated. 


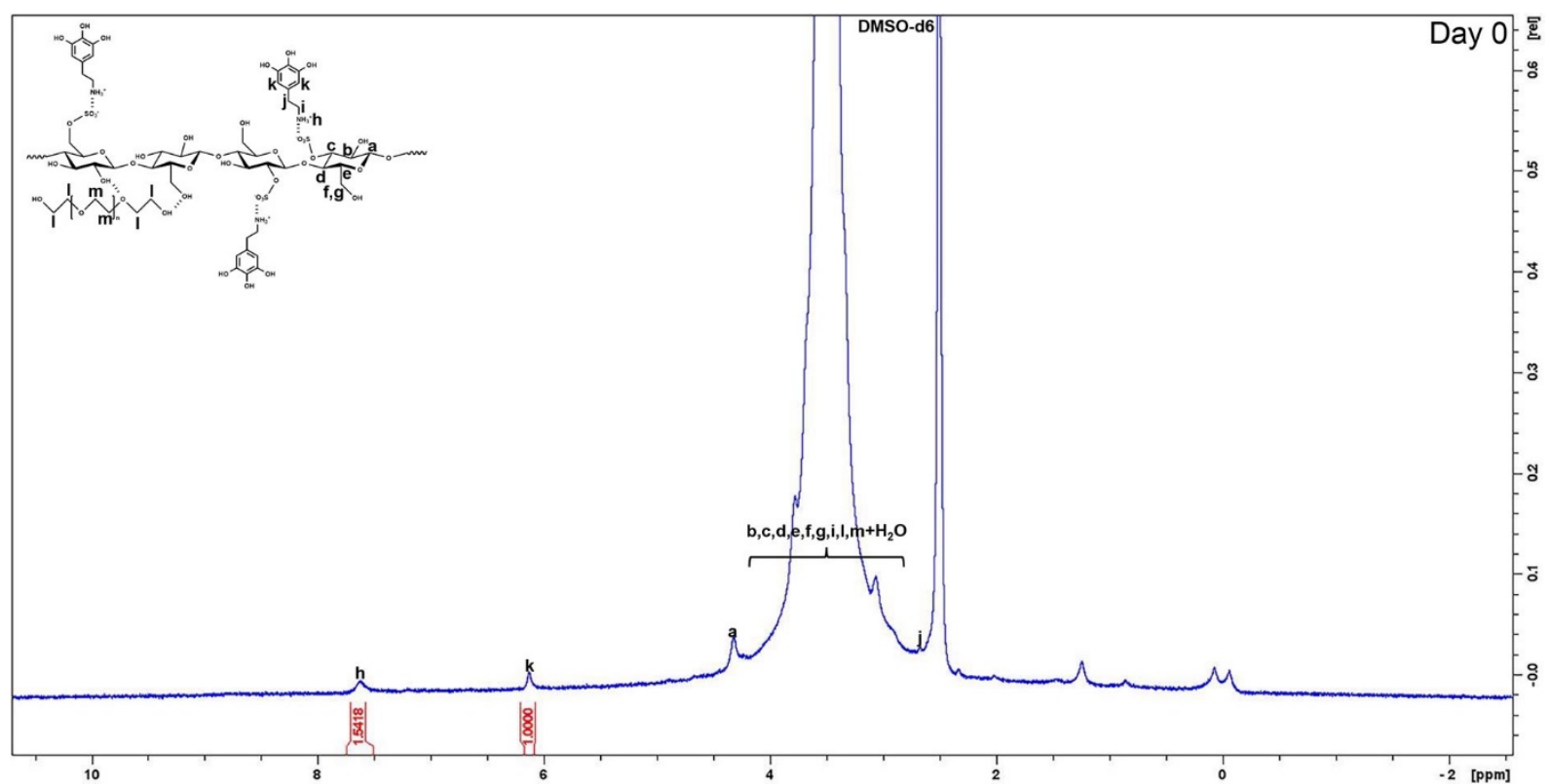

Figure S13. Proton NMR of CNC/TOPA/PEG suspension at day 0 after 24 h stirring of CNC/TOPA/PEG suspension after addition of PEG.

The proton ratio between the ' $k$ ' and ' $h$ ' position of TOPA is 1:1.5 at day 0 after $24 \mathrm{~h}$ stirring of CNC/TOPA/PEG suspension after addition of PEG. It has been known that when the oxidative polymerization of gallol group occurs, the one proton next to the hydroxyl group of gallol is removed $^{[4]}$, leading to proton ration change between the ' $k$ ' and ' $h$ ' position of TOPA from 1:1.5. Therefore, this result indicates that TOPA exist as in the pure compound state at day 0 in the suspension. 


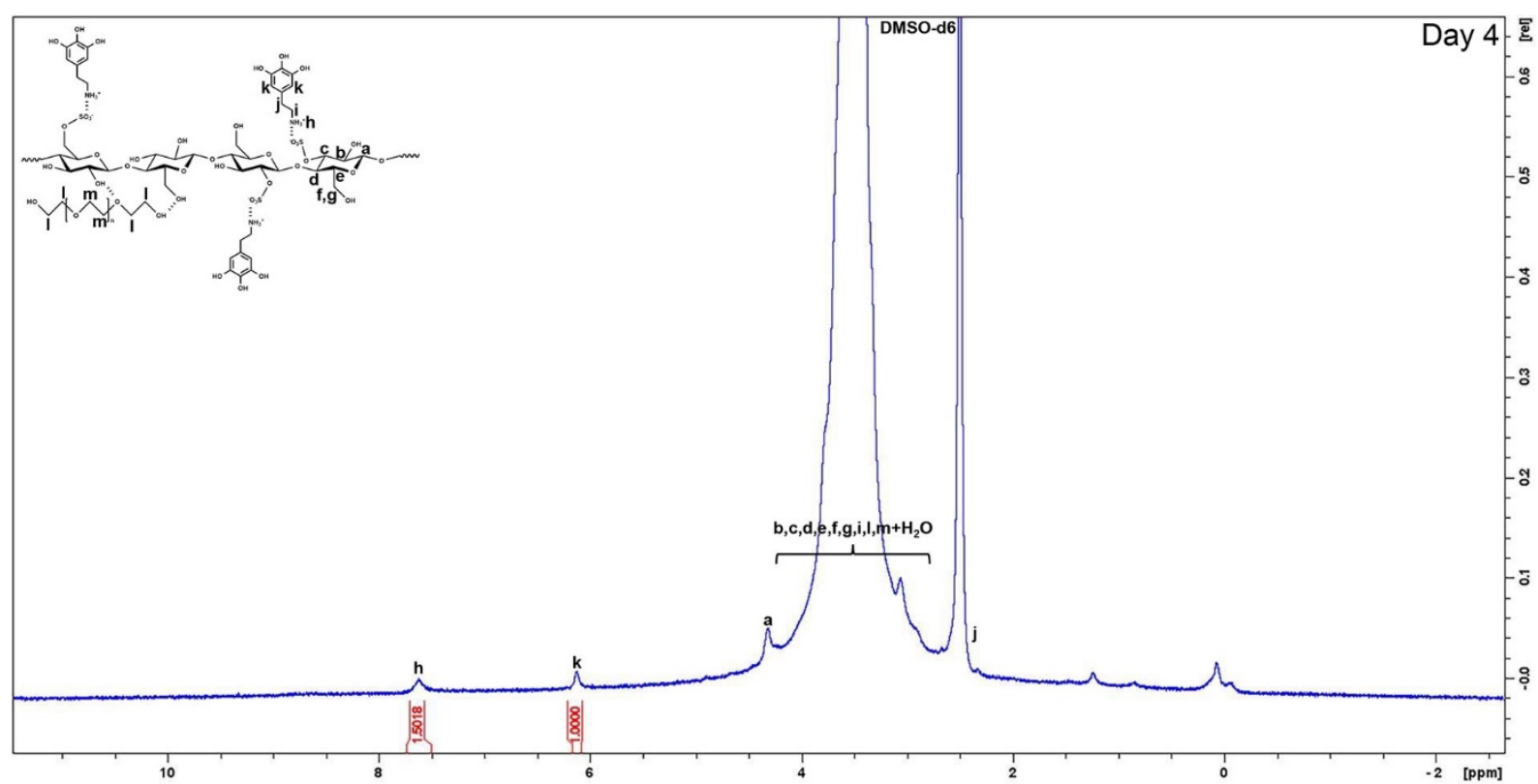

Figure S14. Proton NMR of CNC/TOPA/PEG suspension at day 4 after 24h stirring of CNC/TOPA/PEG suspension after addition of PEG.

The proton ratio between the ' $k$ ' and ' $h$ ' position of TOPA is 1:1.5 at day 4 after 24 h stirring of CNC/TOPA/PEG suspension after addition of PEG, showing that TOPA exist as in the pure compound state at day 4 in the suspension. This result is consistent with the obtained UV-vis spectra (Figure S6) and implies that the TOPA moiety in the CNC/TOPA/PEG film inside of the capillary exist as in the pure compound state. 

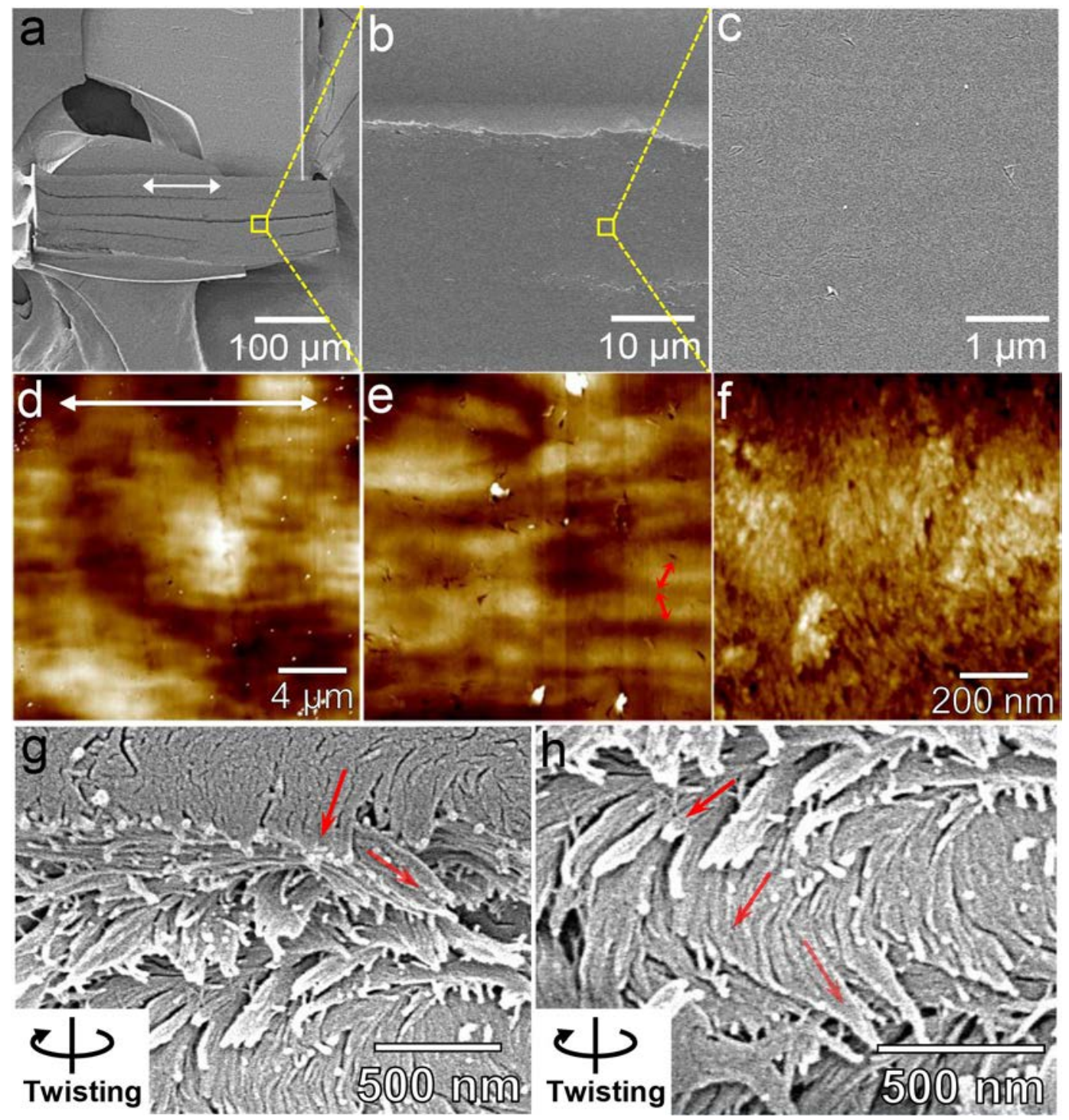

Figure S15. (a-c) SEM top-view, (d-e) AFM, and (g, h) SEM cross-sectional images of CNC: (g) at the surface and (h) inside of capillary. The SEM images depicts that crack is formed in the CNC film (Fig. S10a). Obtained SEM and AFM images presents that herringbone structure at the interface between the capillary and composite over long-range (Figs. S10b, S10c, S10d-e). Crosssectional SEM images reveals that helix axis asymmetry-breaking also occurs (Figs. 10g and 10h); from horizontally organized left-handed chiral organization along the wide capillary tube surface (perpendicular helix axis to the wide surface of capillary tube) to vertical organization inside of capillary (parallel helix axis to the wide surface of capillary tube). 


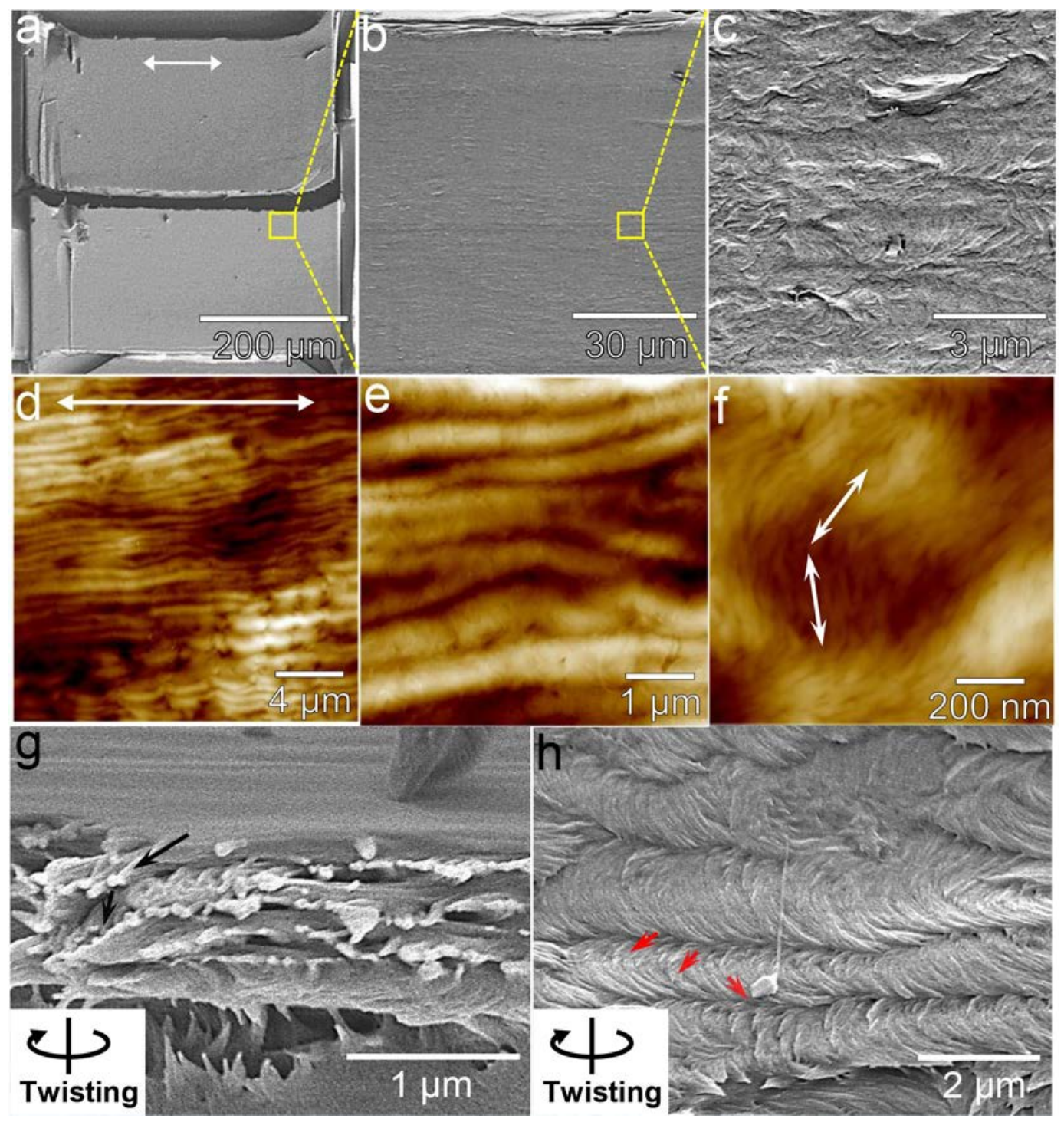

Figure S16. (a-c) SEM top-view, (d-e) AFM, and (g, h) SEM cross-sectional images of CNC/PEG: (g) at the surface and (h) inside of capillary. The SEM images shows that crack is formed in the film (Fig. S12a). The SEM and AFM images illustrates that herringbone structure at the interface between the capillary and composite over long-range (Figs. S12b, S12c, S12d-e). Cross-sectional SEM images reveals that helix axis orientation change also occurs (Figs. S12g and S12h). 


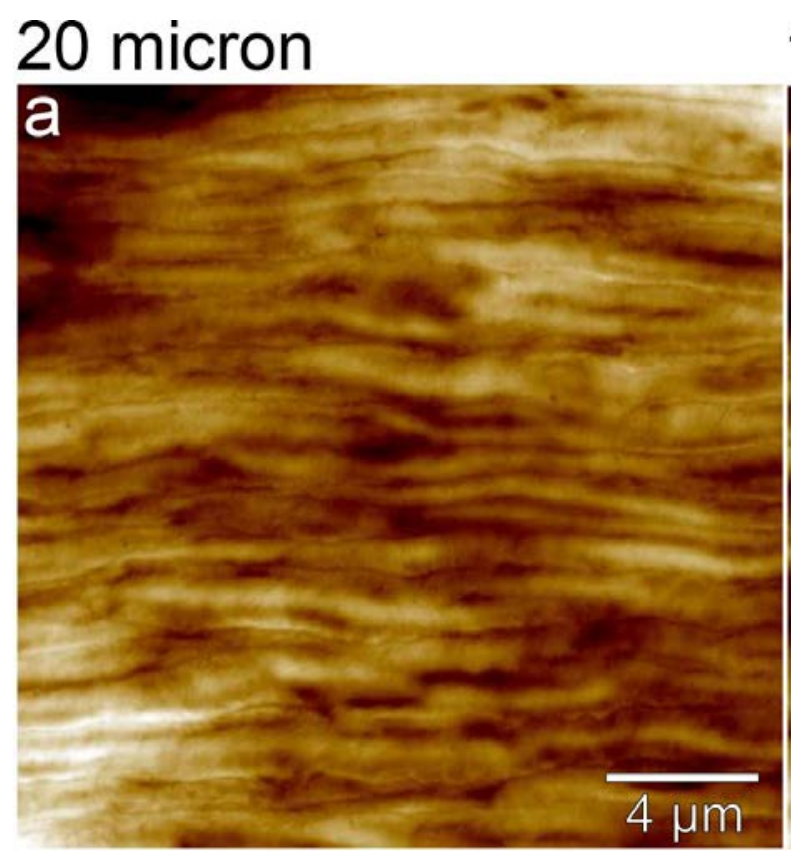

\section{0 micron}

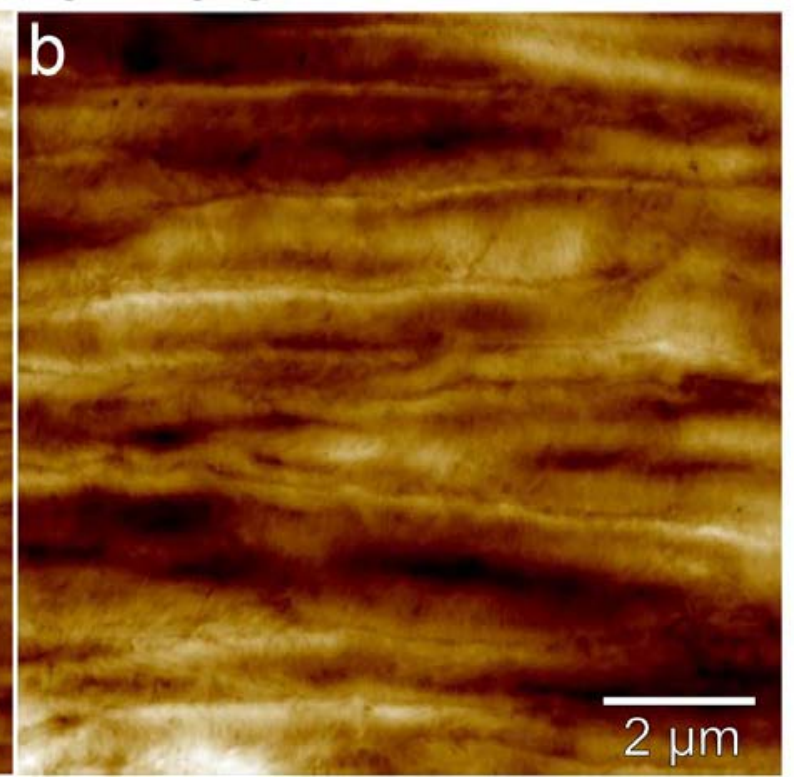

Figure S17. AFM topography images of CNC/TOPA/PEG. (a) $20 \mu \mathrm{m}$ x $20 \mu \mathrm{m}$ and (b) $10 \mu \mathrm{m} x$ $10 \mu \mathrm{m}$, respectively. Achieved AFM images depict that CNC/TOPA/PEG composite is highly uniformly organized as herringbone structure at the interface between the capillary and composite over long-range under capillary confinement. 


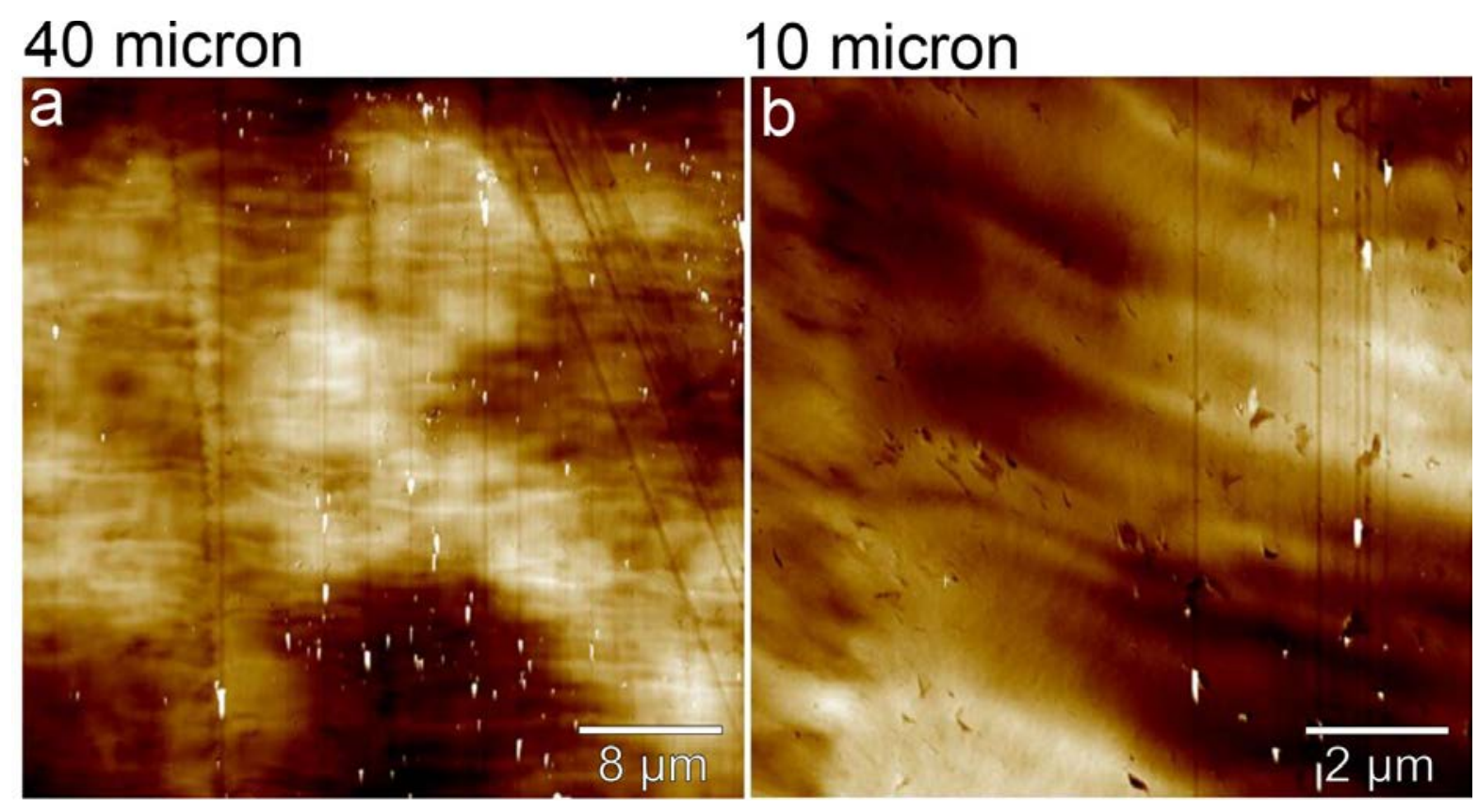

Figure S18. AFM topography images of CNC. (a) $40 \mu \mathrm{m} \times 40 \mu \mathrm{m}$ and (b) $10 \mu \mathrm{m} \times 10 \mu \mathrm{m}$, respectively. Achieved AFM images illustrates that CNCs are uniformly organized as herringbone structure at the interface between the capillary and CNCs over long-range under capillary confinement. 

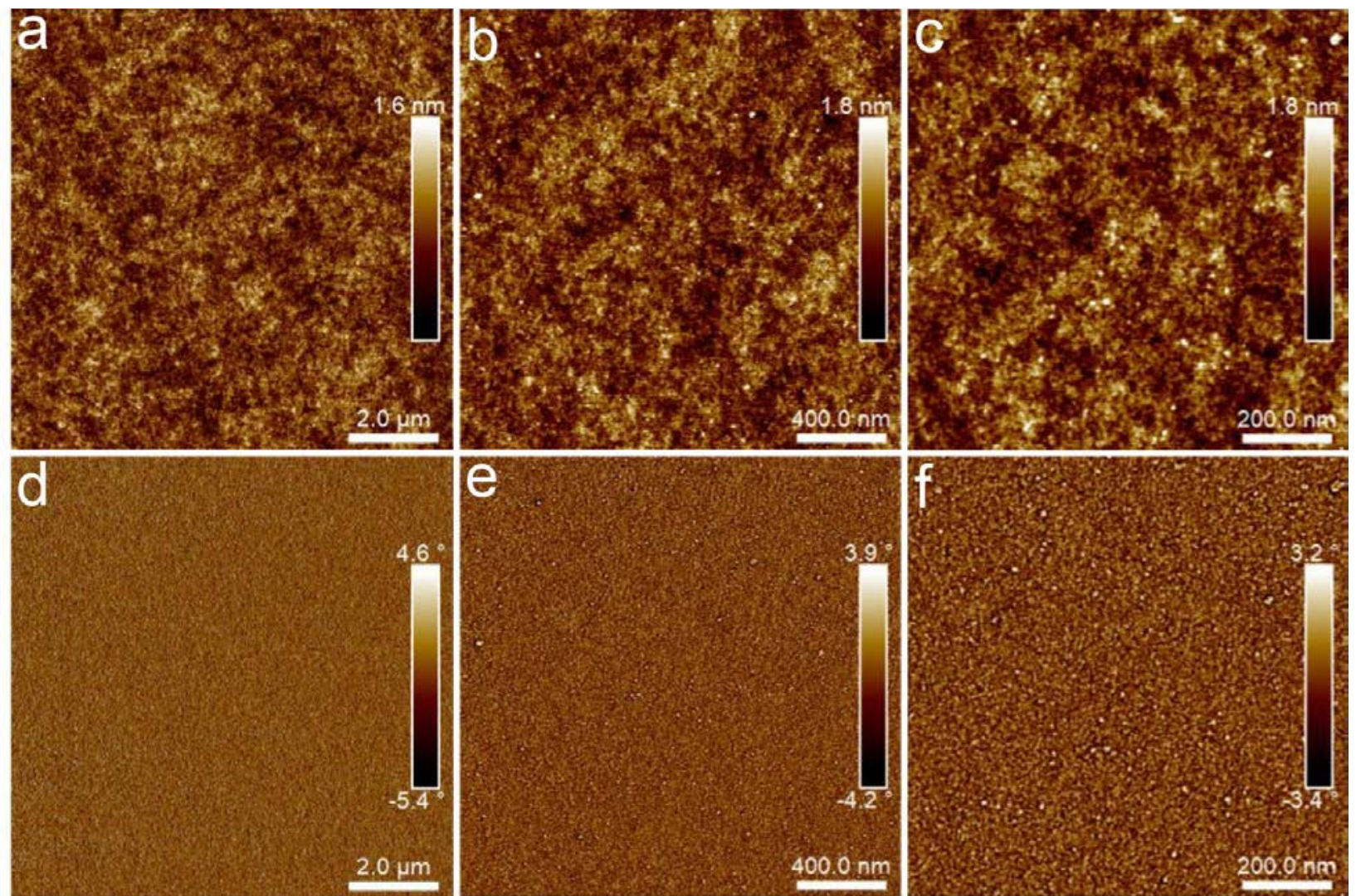

Figure S19. AFM (a-c) topography and (d-e) corresponding phase images of inside surface of empty rectangular capillary. (a, d) $10 \mu \mathrm{m} \times 10 \mu \mathrm{m}$, (b, e) $2 \mu \mathrm{m} \times 2 \mu \mathrm{m}$, (c, f) $1 \mu \mathrm{m} \times 1 \mu \mathrm{m}$, respectively. AFM images present the smooth and uniform inside surface of empty rectangular capillary without any textures. 


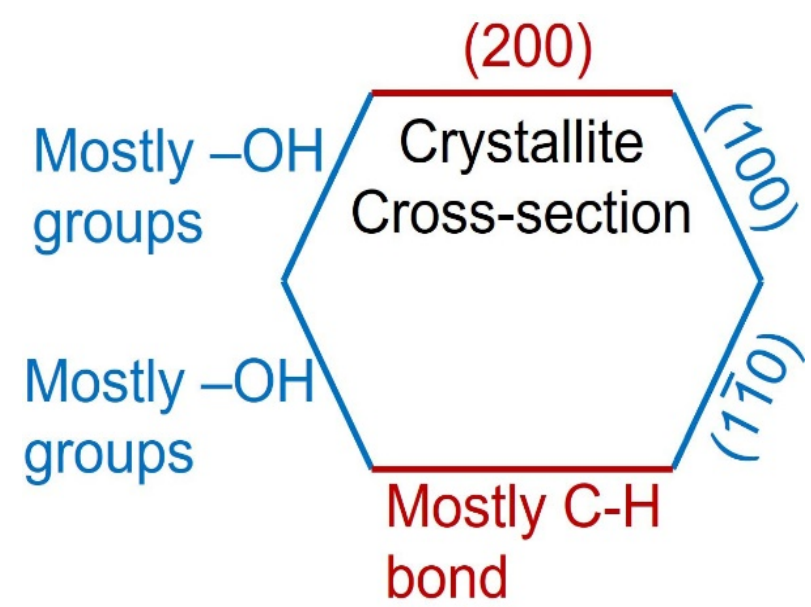

Figure S20. The crystallite structure of CNCs. ${ }^{7}$ The crystallite illustrates up to three kinds of surfaces corresponding to the lattice planes (110), (11̄0), and (200). The (200) plane shows mostly $\mathrm{C}-\mathrm{H}$ bonds, whereas the (110) and (110) planes are rich in hydroxyl groups. ${ }^{7}$ 

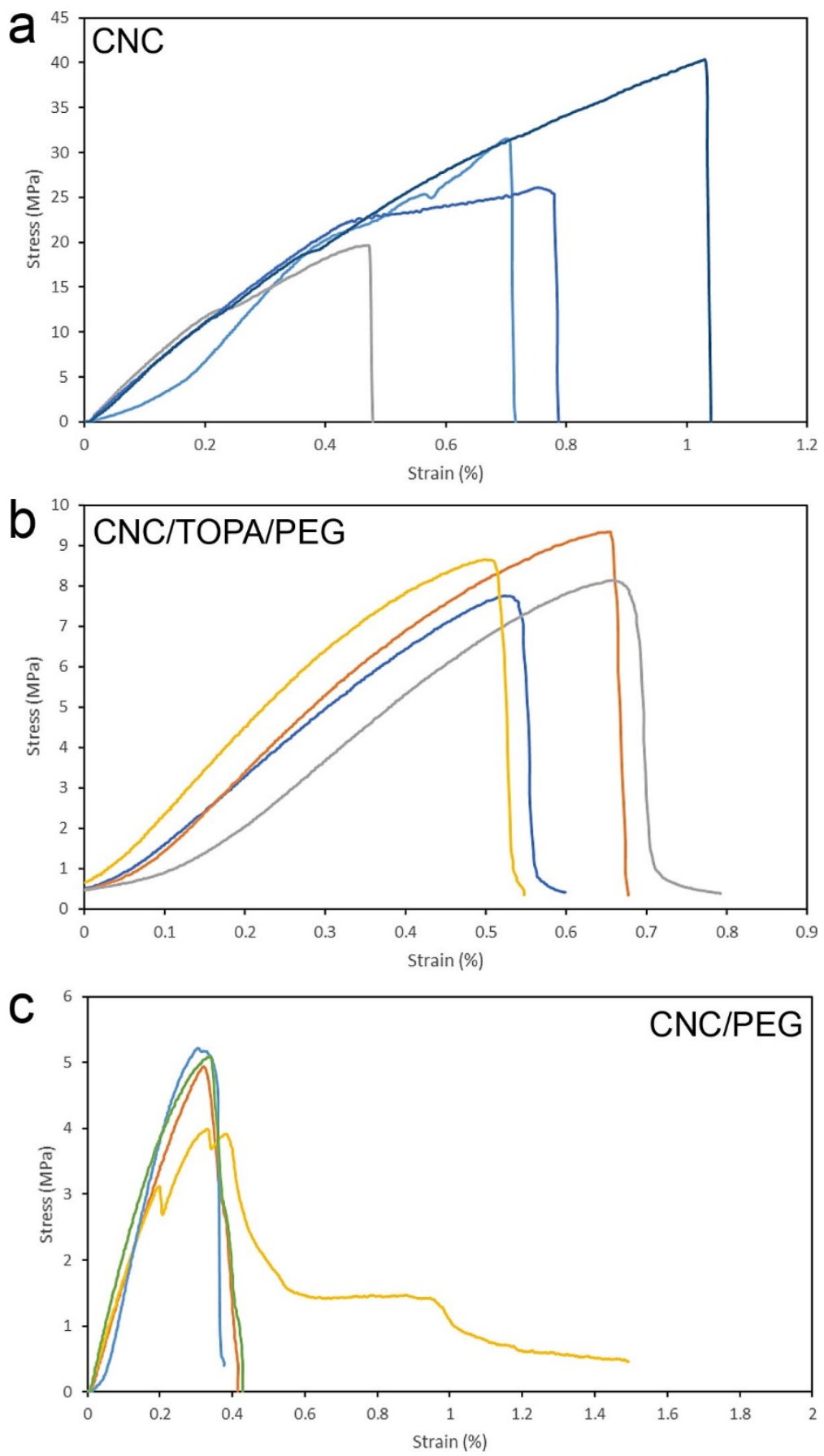

Figure S21. Stress-strain curves of (a) CNC, (b) CNC/TOPA/PEG, (c) CNC/PEG, respectively. 

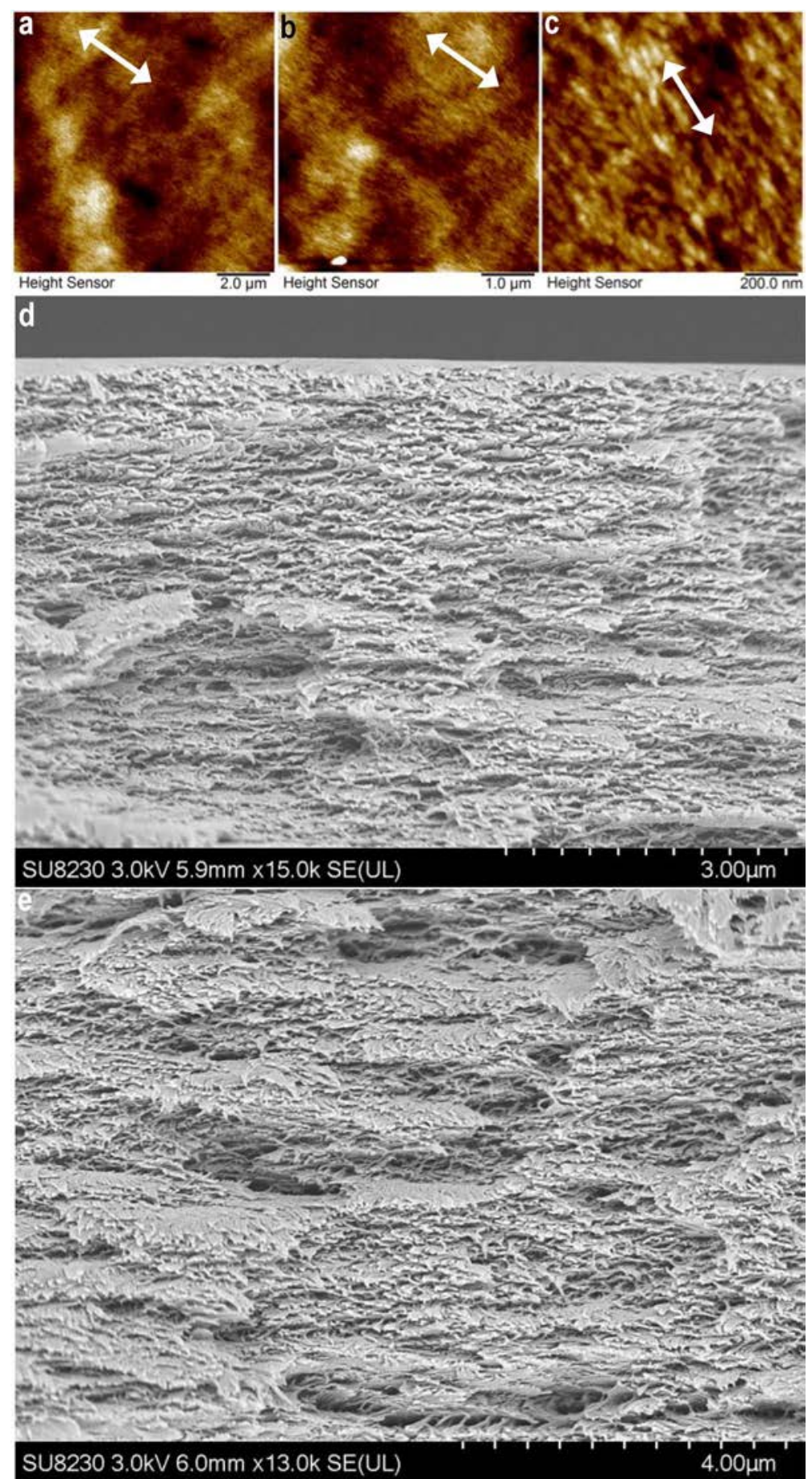

Figure S22. (a-c) Topography AFM and (d, e) cross-sectional SEM images of solution cast CNC film. The AFM images show the nematic structure and SEM images represent the chiral structure of the CNCs in the film. Overall, the film display the horizontally organized left-handed chiral nematic organization along the Petri dish surface (perpendicular helix axis to the surface of Petri dish). 


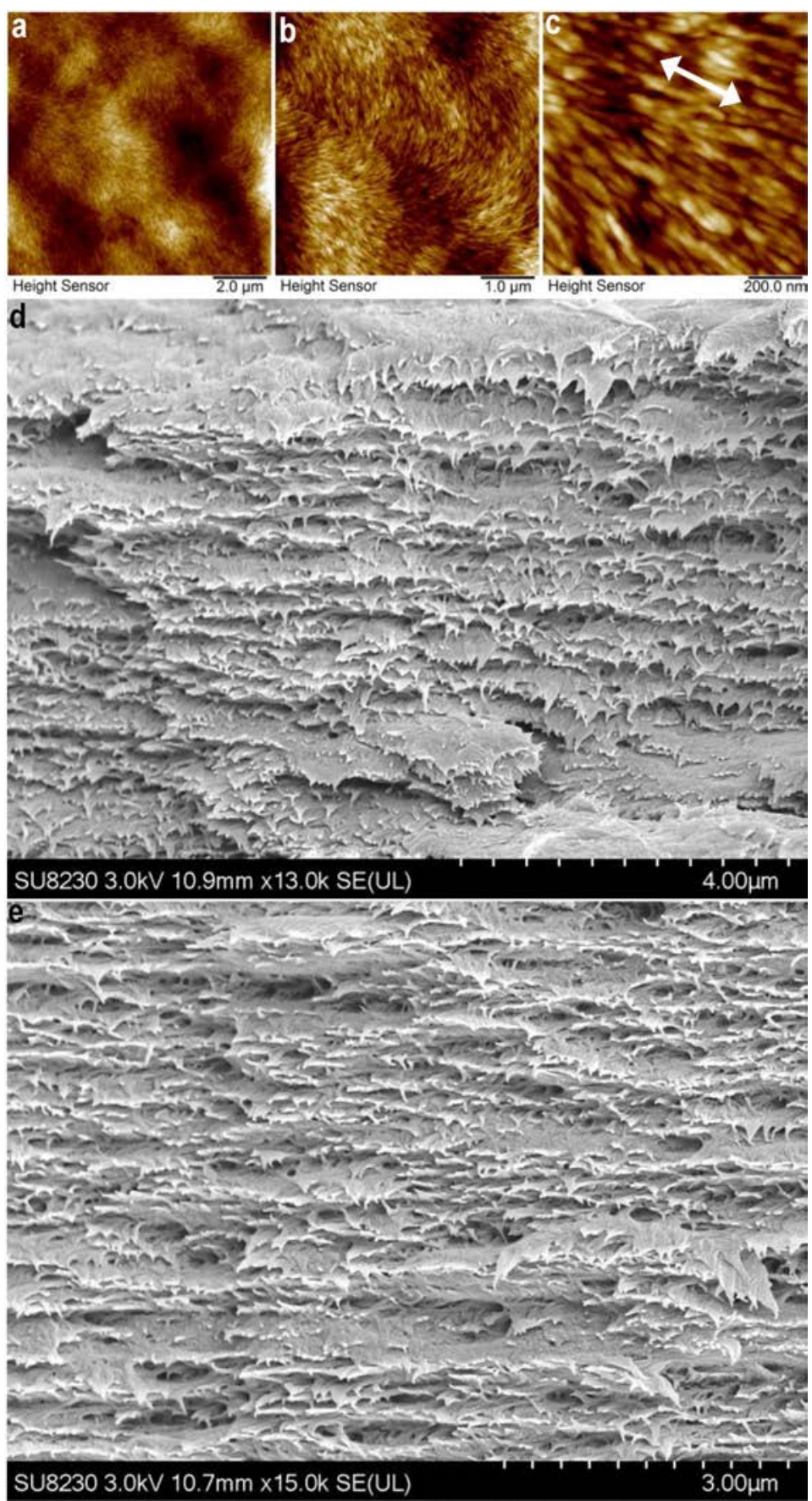

Figure S23. (a-c) Topography AFM and (d, e) cross-sectional SEM images of solution cast CNC/PEG film. The AFM images display the nematic structure and SEM images exhibit the chiral structure of the CNCs in the film. Overall, the film demonstrates the horizontally organized lefthanded chiral nematic organization along the Petri dish surface (perpendicular helix axis). 

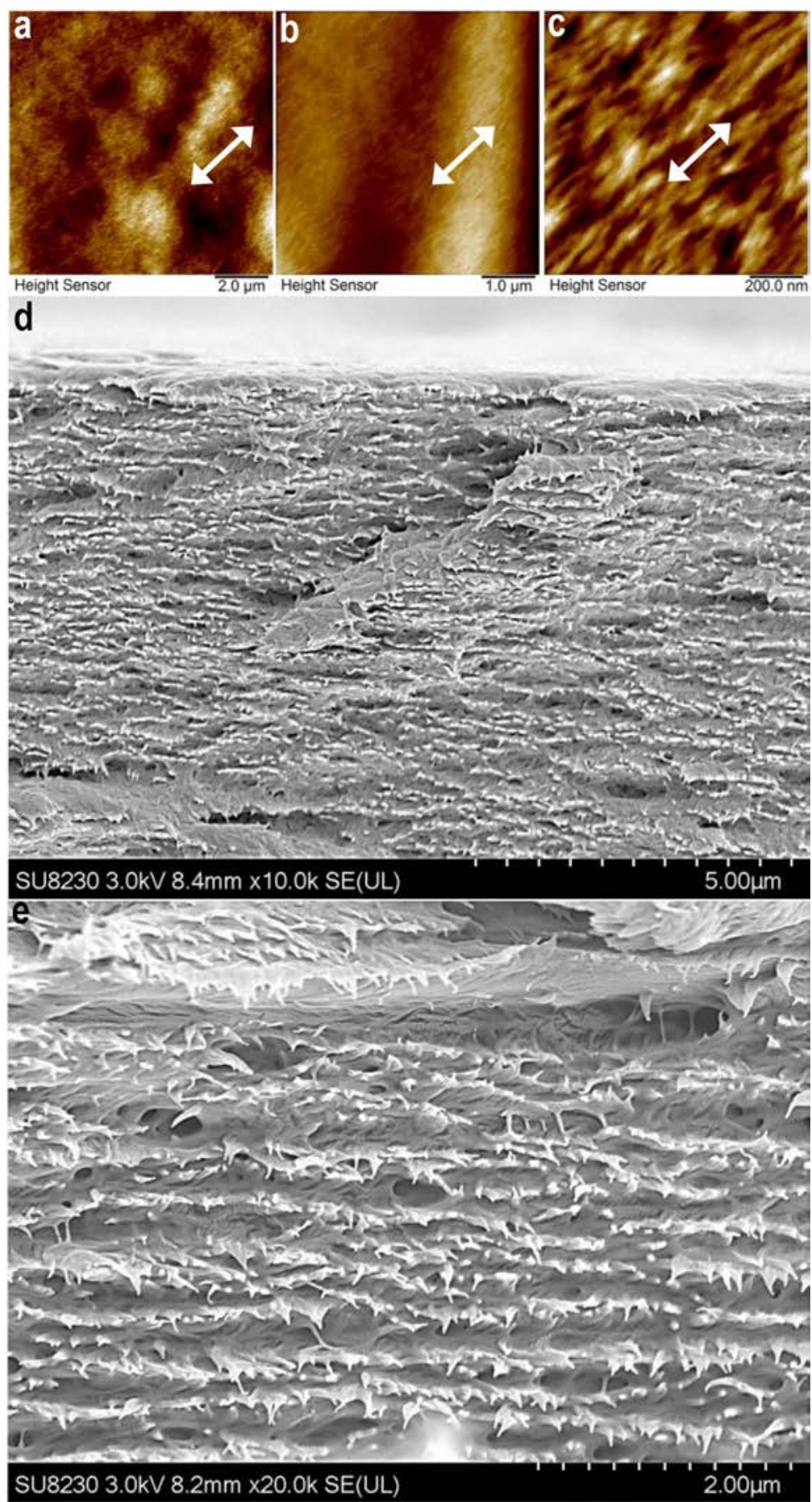

Figure S24. (a-c) Topography AFM and (d, e) cross-sectional SEM images of solution cast CNC/TOPA/PEG film. The AFM images represent the nematic structure and SEM images depict the chiral structure of the CNCs in the film. Overall, the film exhibits the horizontally organized left-handed chiral nematic organization along the Petri dish surface (perpendicular helix axis). 


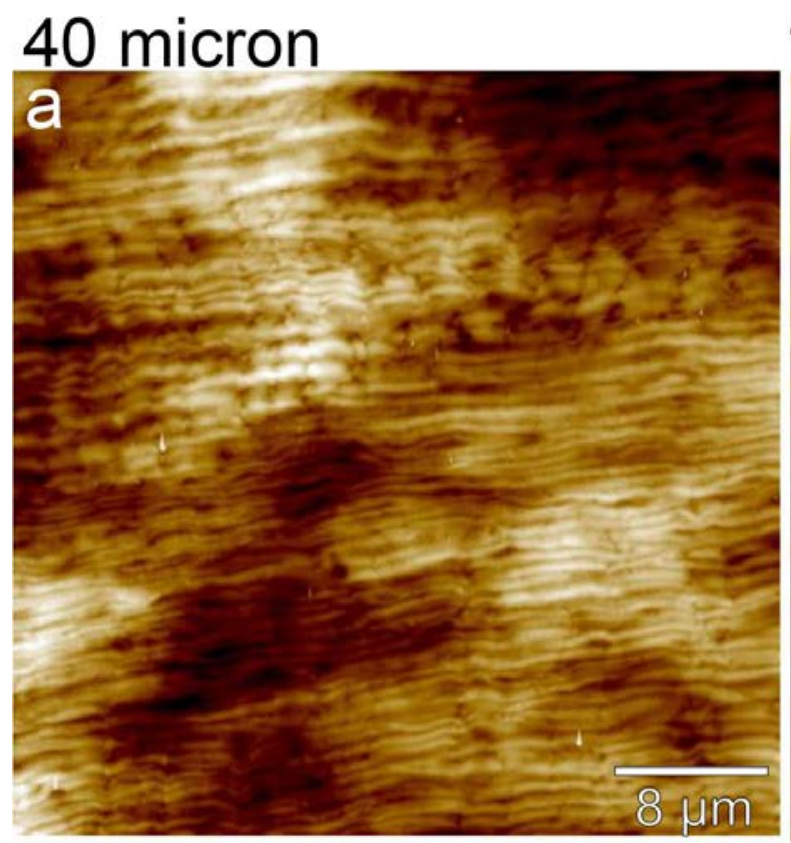

\section{0 micron}

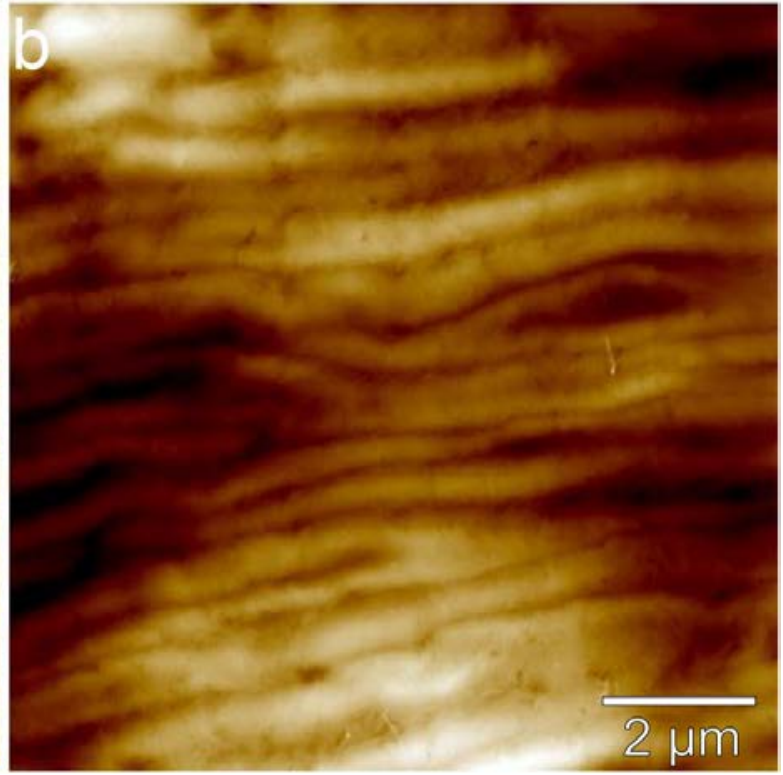

Figure S25. AFM topography images of CNC/PEG films. (a) $40 \mu \mathrm{m} \times 40 \mu \mathrm{m}$ and (b) $10 \mu \mathrm{m} \times 10$ $\mu \mathrm{m}$, respectively. AFM images depict that CNC/PEG composite is uniformly self-assembled as herringbone structure at the interface between the capillary and composite over long-range under capillary confinement. 


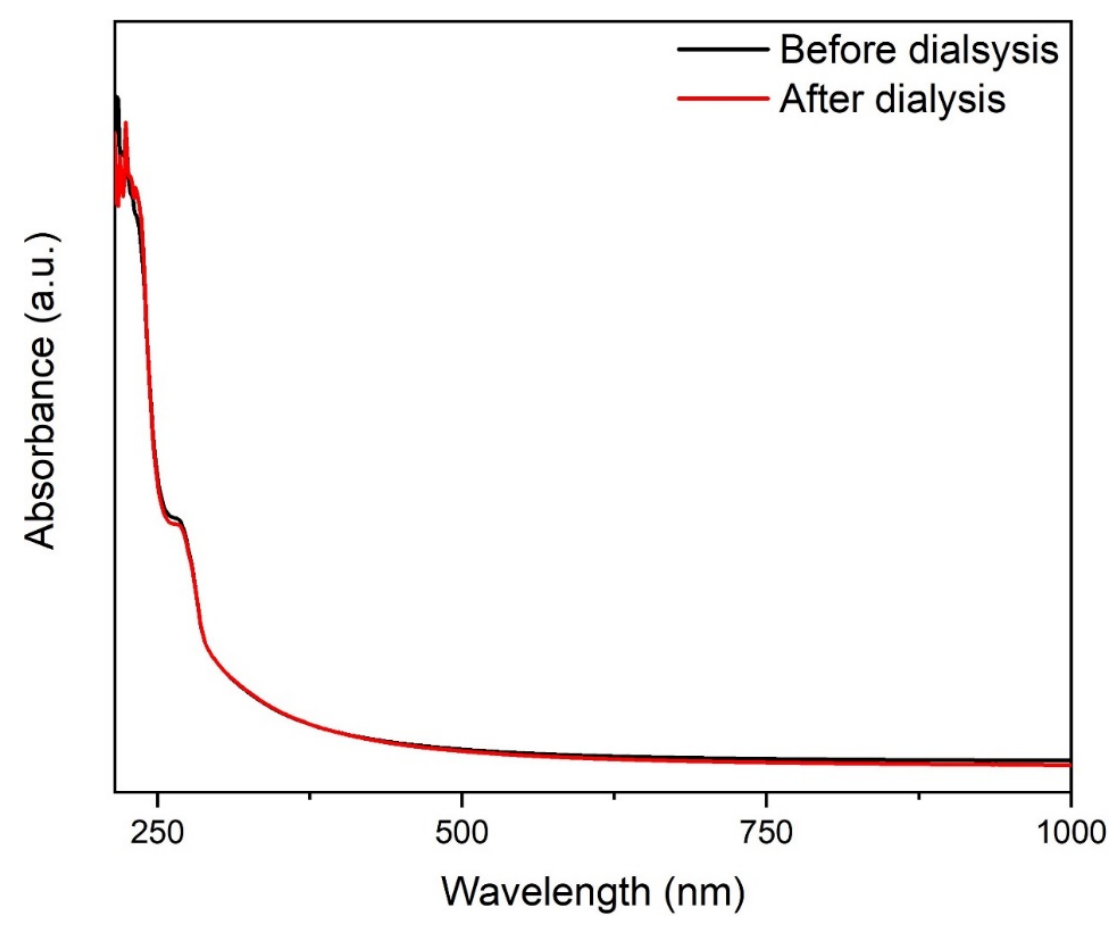

Figure S26. UV-vis spectra of CNC/TOPA/PEG suspension before and after dialysis. 


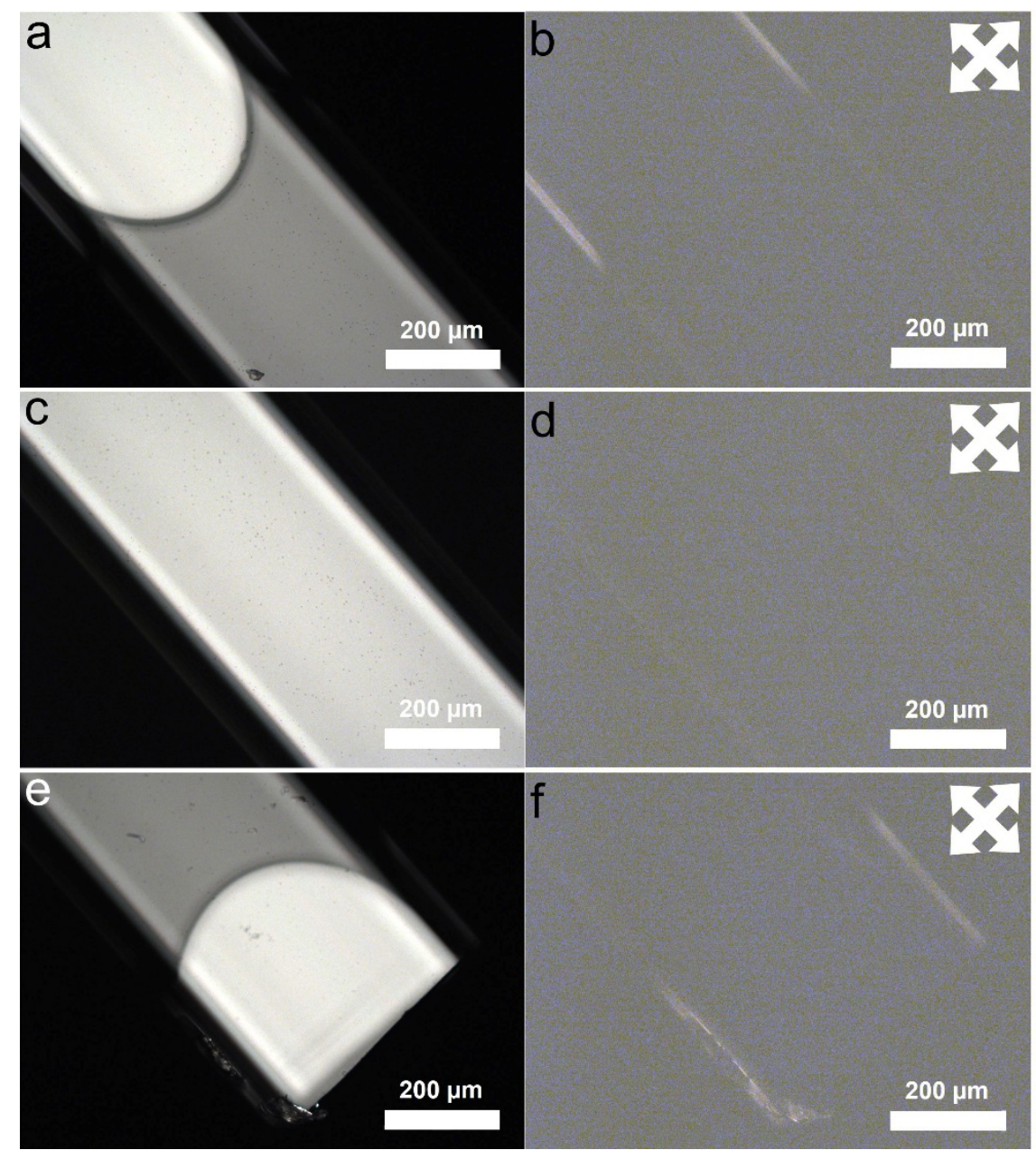

Figure S27. (a, c, e) Unpolarized and (b, d, e) cross-polarized optical microscope images of CNC/TOPA/PEG suspension in capillary tube. (a, e) top, (c, d) middle, and (e, f) bottom (filling) part of capillary tube. The CNC/TOPA/PEG solute and CNC component concentration in the filled CNC/TOPA/PEG suspension are $1.29 \mathrm{wt} \%$ and $0.82 \mathrm{wt} \%$, respectively. The images were immediately taken after filling the CNC/TOPA/PEG suspension which is freshly prepared by performing 24h stirring of PEG with CNC/TOPA. 

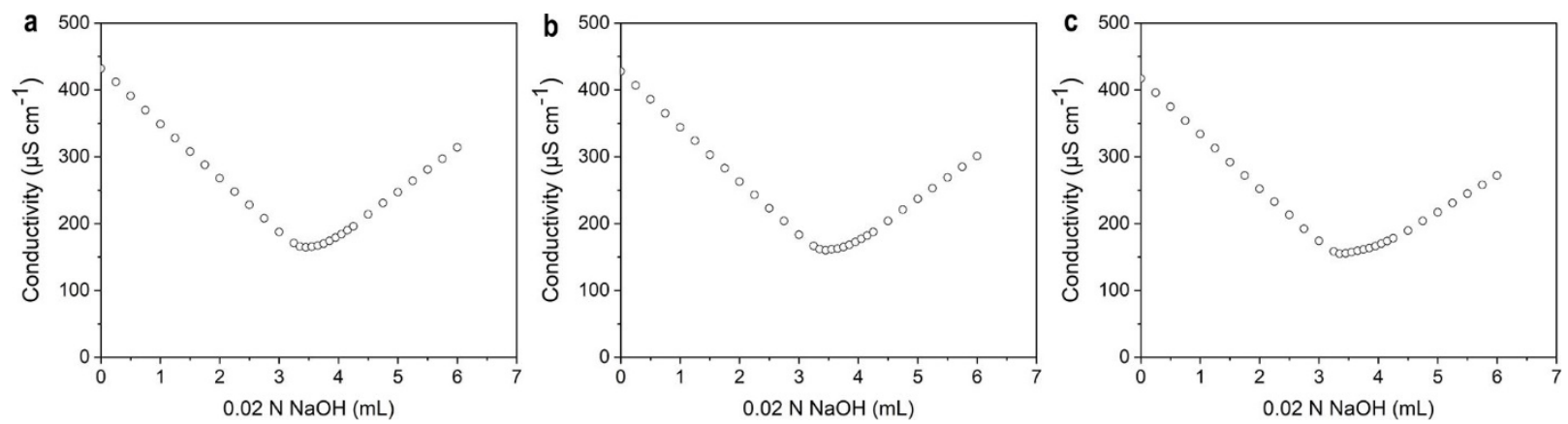

Figure S28. (a-c) Conductometric titration curves for the CNC suspension. 
Table S1. Mechanical properties of CNC/TOPA/PEG, CNC/PEG, and CNC films. ${ }^{\text {a }}$

\begin{tabular}{|c|c|c|c|c|}
\hline Sample & $\begin{array}{c}\text { Young's } \\
\text { modulus } \\
\text { (GPa) }\end{array}$ & $\begin{array}{c}\text { Toughness (kJ m- } \\
\mathbf{3})\end{array}$ & $\begin{array}{c}\text { Ultimate tensile } \\
\text { strength } \\
\text { (MPa) }\end{array}$ & Elongation (\%) \\
\hline $\begin{array}{c}\text { CNC/TOPA/ } \\
\text { PEG }\end{array}$ & $1.8 \pm 0.2$ & $30 \pm 4$ & $8.5 \pm 0.6$ & $0.7 \pm 0.1$ \\
\hline CNC/PEG & $1.8 \pm 0.2$ & $15 \pm 5$ & $4.8 \pm 0.5$ & $0.7 \pm 0.5$ \\
\hline CNC & $4.5 \pm 0.6$ & $140 \pm 70$ & $29 \pm 8$ & $0.8 \pm 0.2$ \\
\hline
\end{tabular}

${ }^{a}$ Measurements have been conducted for freely standing films prepared by evaporating water in the suspension in the Petri dish.

\section{References}

${ }^{1}$ Zhou, H.; Chen, Y.; Plummer, C. M.; Huang, H.; Chen, Y. Facile and Efficient Bromination of HydroxylContaining Polymers to Synthesize Well-Defined Brominated Polymers. Polym. Chem. 2017, 8, 21892196.

${ }^{2}$ King, A. W.; Mäkelä, V.; Kedzior, S. A.; Laaksonen, T.; Partl, G. J.; Heikkinen, S.; Koskela, H.; Heikkinen, H. A.; Holding, A. J.; Cranston, E. D. Liquid-State NMR Analysis of Nanocelluloses. Biomacromolecules 2018, 19, 2708-2720.

${ }^{3}$ Wang, Y.; Park, J. P.; Hong, S. H.; Lee, H. Biologically Inspired Materials Exhibiting Repeatable Regeneration with Self-Sealing Capabilities without External Stimuli or Catalysts. Adv. Mater. 2016, 28, 9961-9968.

${ }^{4}$ Bentley, R. A Fresh Look at Natural Tropolonoids. Nat. Prod. Rep. 2008, 25, 118-138.

${ }^{5}$ Zhan, K.; Kim, C.; Sung, K.; Ejima, H.; Yoshie, N. Tunicate-Inspired Gallol Polymers for Underwater Adhesive: A Comparative Study of Catechol and Gallol. Biomacromolecules 2017, 18, 2959-2966.

${ }^{6}$ Liu, F.; Long, Y.; Zhao, Q.; Liu, X.; Qiu, G.; Zhang, L.; Ling, Q.; Gu, H. Gallol-Containing Homopolymers and Block Copolymers: ROMP Synthesis and Gelation Properties by Metal-Coordination and Oxidation. Polymer 2018, 143, 212-227.

${ }^{7}$ Bruel, C.; Tavares, J. R.; Carreau, P. J.; Heuzey, M.-C. The Structural Amphiphilicity of Cellulose Nanocrystals Characterized from Their Cohesion Parameters. Carbohydr. Polym. 2019, 205, 184-191. 